Article

\title{
Globalization, Country Risks, and Trade in Tourism Services: Evidence from China
}

\author{
Wenwen Zhang and Yi-Bin Chiu *(D) \\ School of Business Administration, Southwestern University of Finance and Economics, Chengdu 611130, China; \\ laura_wenwen@163.com \\ * Correspondence: yibin.chiu@gmail.com
}

Received: 20 June 2020; Accepted: 19 July 2020; Published: 21 July 2020

\begin{abstract}
This study applies the autoregressive distributed lag (ARDL) model to examine the impacts of globalization and country risks on China's tourism service trade over the period 1984-2015. The results reveal that in the long run, globalization has a significant negative impact on tourism service exports and tourism service trade balances, while a significant positive impact on tourism service imports. In the short run, globalization has a significant negative impact on tourism service imports, while a significant positive impact on tourism service exports and trade balances. Country stability could roughly mitigate these negative and positive impacts of globalization on tourism service trade in both the short and long run. Moreover, the speed of adjustment from the short run to long run equilibrium path is relatively fast. These results are important for China's policy makers when formulating a strategy for the development of tourism service trade.
\end{abstract}

Keywords: globalization; country risks; tourism service trade; ARDL

\section{Introduction}

Tourism has become one of the most important industries in the world and its economic impacts are vital for many countries [1,2]. Generally, the development of tourism is considered to positively contribute to economic growth [3,4]. In fact, the World Travel and Tourism Council predicts that the direct contribution of tourism to GDP will maintain an annual growth rate of $3.8 \%$ over the next decade.

International tourism constitutes one of the most significant global trade flows [5] as an increase in international tourism can lead to greater international trade in terms of import demand for foreign goods and services as well as an increase in earnings through exports [6]. In recent years, tourism has been the most important sector in the service trade, accounting for about $25 \%$ of total international trade in services and ranking first in the world. China's trade scale and status in the international tourism market have been remarkable in recent years. The tourism service trade accounts for a high proportion of China's service trade (25.3\% in 2008 and 35.1\% in 2015), representing an important source of the country's service trade income [7]. Before 2008, China's tourism service trade, as one of the trades in service that exhibits a surplus in China, partly offset its trade deficit for many years, playing an important role in increasing foreign exchange income and balancing international payments [8]. However, China entered a deficit period of tourism foreign exchange income in 2009 and this deficit has continued to grow [7]. According to the Yearbook of China Tourism Statistics, the deficit in 2014 grew to US $\$ 113.6$ billion, accounting for $57.7 \%$ of the service trade deficit. The tourism trade deficit has somewhat alleviated relations between China and other countries, but a long-term deficit will harm the interests of the country [8].

Globalization is a comprehensive political, economic, and social phenomenon $[9,10]$ and is considered a determining factor that affects the growth of the tourism industry [11,12]. It is well recognized that globalization could provide favorable opportunities for the development of international 
tourism by motivating more international integration of society and economy [13-15]. Jin et al. [16] claim that tourism development in destinations could be assisted by way of political, social, and cultural exchanges between the generating and destination countries. Economic globalization could decrease barriers that impede the international flow of tourism goods and services. Political globalization could enhance the connections between countries and increase liberalization of the tourism service trade. Trade liberalization has allowed new technologies to be exploited in globalized markets on a worldwide scale [5]. With the development of transportation and information technology, social globalization could decrease the tourists' search cost and make it more feasible and convenient to travel internationally.

Every coin has two sides. Globalization will stimulate competitiveness and create a more complex environment for the tourism industry by facilitating market entry of several stakeholders [13], and could bring some negative effects. Globalization may exacerbate the uneven development of tourism among countries and make developing countries more dependent on developed countries [12]. Some studies also point out that in the context of globalization, the rapid development of international tourism may bring about environmental degradation $[17,18]$. As tourism activities increase, tourism services consumed by international visitors, such as transportation, accommodation, catering, entertainment, and so on, will induce more energy consumption and more pollution, e.g., air and surface water pollution, noise pollution, and soil pollution, etc. However, it should be noted that a skillful tourist management that aimed at serving tourists does not affect natural values and that tourism in the naturally values areas has recently become more and more popular [19].

The country risks, which refer to the external environment uncertainties, also influence passengers' decision-making. Ever since the 911 terrorist attacks in 2001, risks have become an increasingly important consideration when choosing travel destinations. If the perceived level of risk for visitors is too high, then they will avoid international travel $[16,20,21]$ or travel to those countries with lower risks [22]. Especially in the context of globalization, the tourism service trade becomes more sensitive to country risks $[11,23]$. One typical example is how the global spread of the Novel Coronavirus (COVID-19) has heavily affected international travel and tourism service demand [24,25]. The World Tourism Organization [26] estimates that the outbreak of COVID-19 could lead to a 20-30\% drop in international tourist arrivals compared to 2019. In China alone, the downfall of international tourist arrivals is as much as $10.9 \%$ [27]. Some studies propose that COVID-19 will trigger the transformation of the tourism industry on a global scale [28-30], indicating the important role that country risks play in tourism trade under globalization.

The contributions and purposes of this study are as follows. First, existing studies have seldom incorporated globalization into an empirical analysis of tourism's impacts. To the best of our knowledge, no empirical study has paid attention to the impacts of globalization on tourism service trade, including for China. As Hjalager [31] notes, it is worth conceptualizing globalization further from different manifestations and to carry out empirical studies of its impacts on tourism service trade. Therefore, our study incorporates globalization into the empirical analysis framework and analyzes the impacts of globalization on China's tourism service trade.

Second, most of the existing literature has only explored the effects of a certain manifestation of globalization on tourism development by using a single indicator, which cannot provide a comprehensive analysis. To fill this gap, our study adopts multifaceted globalization indices, i.e., an overall index of globalization as well as sub-indices (i.e., economic, social, and political globalization), which enables us to identify which globalization index plays a more important role in China's tourism service trade.

Third, country risks have not been taken seriously in the literature of tourism service trade and therefore, this study considers the impacts of country risks when analyzing the relationship between globalization and China's tourism service trade. Moreover, as the indicators adopted by existing literature did not measure country risks well, in order to improve the situation, we adopted the political 
risk index and composite risk index retrieved from the International Country Risk Guide (ICRG) to measure country risks comprehensively.

The rest of the paper is organized as follows. Section 2 briefly reviews the relevant literature. Section 3 discusses the econometric methods. Sections 4 and 5 presents the data specification and the empirical results, respectively. Section 6 outlines the conclusions and some implications.

\section{Literature Review}

The existing studies have analyzed many determinants of tourism service trade and conclude that history, economy, politics, socio-culture, and attractiveness of a destination are important factors that affect tourism service trade [32-35]. Similarly, studies about China's tourism service trade also conceptually mention these influencing factors $[7,8,36]$.

Globalization may also be an important factor influencing tourism service trade, and regionalism is a representative opposite factor against globalization. Regionalism is leading to the establishment of discrete and increasingly powerful groups of countries that may form regional protection bastions against certain forces of globalization, while globalization is removing economic barriers between countries [37]. By studying the case of Bhutan, Nyaupane and Timothy [38] propose that tourists could be treated unequally under regionalism. Henderson [39] uses the example of the Indonesia-Malaysia-Singapore Growth Triangle and concludes that the impact of regionalism on tourism development remains uncertain. Dredge and Jenkins [40] found that through regionalism, some countries aim to establish strong and attractive tourism destination identities in order to counterbalance the homogenizing influences of globalization.

As for globalization, it has still not been discussed widely in tourism research. Early studies only considered certain manifestations, with exploring the impact of international hotel chains [41,42], commodity chains [43], and airlines [44] on tourism development. Hjalager [31] distinguishes four stages and different manifestations of the tourism industry's globalization and shows that it is undergoing an irrevocable process like many other business systems. Some studies examine the economic impacts of tourism within the macroeconomic context of globalization in the form of increasing trade liberalization [5,45]. Sugiyarto, Blake, and Sinclair [45] use a computable general equilibrium model of the Indonesian economy and find that tourism growth expands the positive effects of globalization and reduces its adverse effects, such as increasing production, improving welfare, and reducing the adverse effects on government deficits and trade balances. On the other hand, some studies address the important effects of globalization in the context of tourism, including economic development (economic globalization), institutional development, the spread of technical knowledge, the development of new markets and products, environmental and sociocultural changes (social globalization), etc. [13-15,46]. The finding of Hidayati [1] supports the notion of the positive effect of globalization regarding the economic growth contribution of tourism. However, Ivanov and Webster [10] find that there is no correlation between globalization and the average per capita economic growth contribution of tourism. Furthermore, by using a sample of 133 countries, Javid and Katircioglu [23] found that economic, social, and political globalization exert a significantly positive impact on tourist arrivals, tourism receipts, and tourism expenditures.

Throughout the tourism-related research, country risks have not been taken seriously, yet the research conducted by Sequeira and Nunes [47] is more prominent. The results of Sequeira and Nunes [47] suggest that country risks are an important determinant of a country's tourism development and policy makers should be aware of the important role of country risks in tourism. Through a case study of the evolution of tourism in the Maldives, Scheyvens [48] proposes that issues of power and politics are crucial to the sustainable development of tourism. Eilat and Einav [49] show a negative correlation between political risks and bilateral visitor flows. Yap and Saha [50] argue that political stability plays an important role in determining destination choices for tourists and discuss political instability as well as the impact of terrorism and corruption on tourism development. Joshi et al. [51] point out that international tourism income is more sensitive to favorable tourism laws and regulations, 
rich natural resources, and cultural heritage, health, and sanitation. Saha et al. [52] present that civil liberties and economic freedom have positive and significant correlations with inbound tourism. When civil liberties (economic freedom) are relatively low, economic freedom (civil liberties) plays a more influential role in inbound tourism. Musavengane et al. [53] indicate that poor governance (political risks) by African governments is harmful to the sustainable development of tourism.

With the deepening of globalization, interactions between countries have increased. The most local developments may come to have enormous global consequences [54]. This means that under globalization, a country's international tourism sector is highly sensitive to country risks. The economic, political, and financial fluctuations of a country (i.e., country risks), such as the financial crisis since 2008 and sociopolitical upheaval in North Africa and the Middle East, could deter tourism consumption and tourism production on a global scale [11]. Based on the above analysis, we can infer that an interaction effect of globalization and country risks on tourism development may exist. There are also a few studies that consider both globalization and risk factors in the field of tourism research. The results of Ivanov and Ivanova [55] suggest that the general business environment (corruption level of a country and globalization) positively affects a destination's tourism competitiveness. While conceptually analyzing the impact of globalization on both the supply side and the demand side of the tourism sector, Song et al. [11] also analyze the impact of business cycles and global crises on tourism and propose that studying the relationship between globalization, risks, and tourism is a future research direction. Alola et al. [56] found that tourism receipts in Turkey could potentially be shocked by uncertainties related to the exchange rate, export trade (globalization), and geopolitical risk.

Of the above literature, no study focuses on the issue of the relationship between globalization, country risks, and tourism service trade. Thus, this study explores the impacts of globalization and country risks on China's tourism service trade.

\section{Methodology}

We specify the basic model of tourism service trade as follows, which is designed to discover the impacts of globalization on tourism service trade:

$$
\operatorname{LnTS}_{t}=a+b \operatorname{LnGLOB} t+c \operatorname{LnRISK} K_{t}+d \operatorname{LnGDP} P_{t}+e \operatorname{LnREER_{t}}+\mu_{t} .
$$

In order to examine the role of country risks on the relationship between globalization and tourism service trade, we modify the basic specification by adding the interaction term to our model as follows:

$$
\operatorname{LnTS}_{t}=a+b \operatorname{LnGLOB} t+c(\operatorname{LnGLOB} * \operatorname{LnRISK})_{t}+d \operatorname{LnGDP} t+e \operatorname{LnREER_{t}}+\mu_{t},
$$

where $\operatorname{LnTS}_{t}$ - the log-transformed tourism service trade, including tourism service exports $\left(\operatorname{LnTSEX} X_{t}\right)$, imports $\left(\operatorname{LnTSIM}_{t}\right)$, and trade balance $(\operatorname{LnTSTB} t)$.

LnTSEX - the log-transformed tourism service exports (\% of service exports), reflecting the exports of services consumed by travelers in a country.

$\operatorname{LnTSIM}_{t}$ - the log-transformed tourism service imports (\% of service imports), reflecting the imports of services consumed by travelers in a country.

$\operatorname{LnTSTB}_{t}$ - the ratio of tourism service exports to tourism service imports.

$L n G L O B_{t}$ - the log-transformed globalization indices, including overall ( $\left.\mathrm{LnOG}_{t}\right)$, economic $\left(L n E G_{t}\right)$, social $\left(L n S G_{t}\right)$, and political $\left(L n P G_{t}\right)$ globalization.

LnRISK $_{t}$ - the log-transformed country risk indices, including composite $(\operatorname{LnCR} t)$ and political $\left(\operatorname{LnPR} R_{t}\right)$ risks.

$\operatorname{LnGDP} P_{t}$-the log-transformed per capita real GDP.

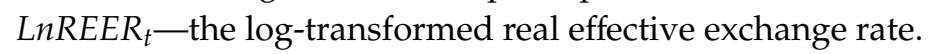

$\mu_{t}$-the white noise error terms. 
Based on the autoregressive distributed lag (ARDL) approach proposed by Pesaran et al. [57], we can reformulate the empirical model as the following Unrestricted Error Correction Model (UECM), containing long-term and short-term information.

$$
\begin{aligned}
& D L n T S_{t}=\delta_{0}+\delta_{1} \operatorname{LnTS}_{t-1}+\delta_{2} \operatorname{LnGLOB}_{t-1}+\delta_{3} \operatorname{LnRISK}_{t-1}+\delta_{4} \operatorname{LnGDP}_{t-1} \\
& +\delta_{5} \operatorname{LnREER}_{t-1}+\sum_{i=1}^{m} b_{i} \operatorname{DLnTS}_{t-i}+\sum_{i=0}^{m} c_{i} \operatorname{DLnGLOB}{ }_{t-i} \\
& +\sum_{i=0}^{m} d_{i} D L n R I S K_{t-i}+\sum_{i=0}^{m} e_{i} D L n G D P_{t-i}+\sum_{i=0}^{m} f_{i} D L n R E E R_{t-i}+\varepsilon_{t} \\
& \operatorname{DLnTS}_{t}=\delta_{0}+\delta_{1} \operatorname{LnTS}_{t-1}+\delta_{2} \operatorname{LnGLOB}_{t-1}+\delta_{3}\left(\operatorname{LnGLOB} * \operatorname{LnRISK}_{t-1}\right. \\
& +\delta_{4} \operatorname{LnGDP}_{t-1}+\delta_{5} \operatorname{LnREER}_{t-1}+\sum_{i=1}^{n} b_{i} \operatorname{DLnTS}_{t-i}+\sum_{i=0}^{n} c_{i} \operatorname{DLnGLOB}_{t-i} \\
& +\sum_{i=0}^{n} d_{i} D(\operatorname{Ln} G L O B * \operatorname{LnRISK})_{t-i}+\sum_{i=0}^{n} e_{i} D \operatorname{LnGDP} P_{t-i} \\
& +\sum_{i=0}^{n} f_{i} D L n R E E R_{t-i}+\varepsilon_{t}
\end{aligned}
$$

where $D$ is the first difference operators.

To examine whether tourism service trade and other independent variables are cointegrated, this study applied a bounds test based on the F-statistics. We denote the F-statistic of the test, which we normalized on the dependent variables, by $\mathrm{f}($.$) . The null hypothesis of no cointegration$ $\left(H_{0}: \delta_{1}=\delta_{2}=\delta_{3}=\delta_{4}=\delta_{5}=0\right)$ was tested against the alternative hypothesis of $\left(H_{1}: \delta_{1} \neq 0\right.$, $\delta_{2} \neq 0, \delta_{3} \neq 0, \delta_{4} \neq 0, \delta_{5} \neq 0$ ) by using the F-test. Pesaran et al. [57] tabulate two sets of new critical values; one set assumes all variables are I(1), i.e., upper bound critical values and another one assumes all variables are I(0), i.e., lower bound critical values. If the calculated F-statistic becomes greater than the I(1), then the null of no cointegration is rejected, indicating that a longer-term relation exists. Contrarily, co-integration does not exist if the F-statistic is less than $\mathrm{I}(0)$. Thus, no decision can be made when the F-statistic is between $\mathrm{I}(0)$ and $\mathrm{I}(1)$.

We lastly obtained the short-run dynamic parameters by estimating an error correction model associated with the long-run estimates. The vector error correction model is specified as follows:

$$
\begin{aligned}
& \operatorname{DLnTS}_{t}=a+\sum_{i=1}^{m} b_{i} D \operatorname{LnTS}_{t-i}+\sum_{i=0}^{m} c_{i} D L n G L O B_{t-i}+\sum_{i=0}^{m} d_{i} D \operatorname{LnRISK}_{t-i} \\
& +\sum_{i=0}^{m} e_{i} D \operatorname{LnGDP} P_{t-i}+\sum_{i=0}^{m} f_{i} D L n R E E R_{t-i}+\gamma E C T_{t-1}+\omega_{t} \\
& \operatorname{DLnTS}_{t}=a+\sum_{i=1}^{n} b_{i} \operatorname{DLnTS}_{t-i}+\sum_{i=0}^{n} c_{i} \operatorname{DLnGLOB}{ }_{t-i} \\
& +\sum_{i=0}^{n} d_{i} D(\operatorname{LnGLOB} * \operatorname{LnRISK})_{t-i}+\sum_{i=0}^{n} e_{i} D \operatorname{LnGDP} P_{t-i} \\
& +\sum_{i=0}^{n} f_{i} D \operatorname{LnREER} R_{t-i}+\gamma E C T_{t-1}+\omega_{t}
\end{aligned}
$$

where $b_{i}, c_{i}, d_{i}, e_{i}$, and $f_{i}$ are the short-run dynamic coefficients of the model's convergence to equilibrium and $\gamma$ is the speed of adjustment.

\section{Data Specification}

This study uses annual time-series data covering the period from 1984 to 2015 . We take the data for tourism service exports (TSEX), tourism service imports (TSIM), tourism service trade balance (TSTB), real GDP per capita (GDP, constant 2010 US\$), and real effective exchange rate (REER, $2010=100$ ) from the World Bank's World Development Indicators (WDI) database.

The globalization indices are from the Konjunkturforschungsstelle (KOF) database of the Swiss Economic Institute, which is developed by Dreher [9] and updated in Dreher et al. [58]. The KOF 
globalization index comprises an overall globalization index $(O G)$ and three separate component indices for economic $(E G)$, social $(S G)$, and political $(P G)$ globalization. The overall globalization index was obtained by a weighted average of $36 \%, 37 \%$, and $27 \%$ of the economic, social, and political globalization indices, respectively. All globalization indices ranged from 0 to 100 . Higher values of indices denoted greater levels of globalization for the country.

We adopted the ICRG risk data constructed by the PRS Group. The ICRG risk indices denote a country's political stability, the ability to finance its official, commercial, and trade debt obligations, and its economic strengths and weaknesses on a comparable basis. We selected political risk index $(P R)$ and composite risk index $(C R)$ to measure country risks. Political risk index and composite risk index both were scored from 0 to 100 . A higher risk rating implied a lower country risk faced by the country. All variables were taken in natural logarithmic form.

Tables 1 and 2 report the descriptive statistics and correlations of the variables. Table 1 shows that on average, the ratio of China's tourism service exports to its service exports and the ratio of China's tourism service imports to its service imports were, respectively, $29.96 \%$ and $23.34 \%$ during the period 1982-2015. On average, during this period, China seems to have little tourism service trade surplus. However, after 2009, China's tourism service imports rapidly increase and its increasing speed is faster than tourism service exports (see Figure 1). This leads to a continuous and rapid expansion of tourism service trade deficits. The tourism service trade deficits enlarge from US $\$ 4$ billion in 2009 to US $\$ 205$ billion in 2015, which could be because the living standards and qualities of Chinese people improve as China's economy grows, thus raising the demand for outbound tourism. According to Pearson's correlation in Table 2, globalization has significantly negative effects on tourism service exports and positive effects on tourism service imports, suggesting that globalization has restricted the exports of China's tourism service, while it has promoted its imports. Moreover, there is a significantly negative relationship between globalization and tourism service trade balances.

Table 1. Descriptive statistics of variables in levels over the period 1984-2015.

\begin{tabular}{cccccc}
\hline Variable & Obs & Mean & Std. Dev. & Min & Max \\
\hline LnTSEX & 32 & 3.40 & 0.28 & 2.98 & 3.90 \\
LnTSIM & 32 & 3.15 & 0.51 & 1.66 & 4.05 \\
LnTSTB & 32 & 0.25 & 0.70 & -1.02 & 1.83 \\
LnOG & 32 & 3.84 & 0.32 & 3.20 & 4.13 \\
LnEG & 32 & 3.77 & 0.18 & 3.33 & 3.99 \\
LnSG & 32 & 3.45 & 0.65 & 2.18 & 4.01 \\
LnPG & 32 & 4.20 & 0.26 & 3.70 & 4.44 \\
LnCR & 32 & 4.28 & 0.09 & 4.05 & 4.39 \\
LnPR & 32 & 4.17 & 0.07 & 4.03 & 4.32 \\
LnGDP & 32 & 7.48 & 0.80 & 6.18 & 8.78 \\
LnREER & 32 & 4.28 & 0.09 & 4.05 & 4.39 \\
\hline
\end{tabular}

Notes: Std. Dev., Max., and Min. are the abbreviations for standard deviation, maximum, and minimum, respectively. All variables are in natural logarithms.

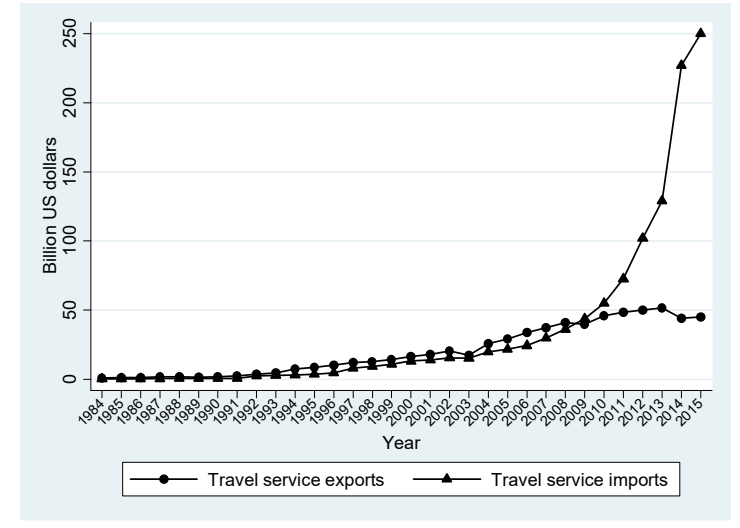

Figure 1. Travel service exports and travel service imports for China, 1984-2015. 
Table 2. Pearson's coefficients of the correlation matrix for variables.

\begin{tabular}{|c|c|c|c|c|c|c|c|c|c|c|c|}
\hline & LnTSEX & LnTSIM & LnTSTB & LnOG & LnEG & LnSG & LnPG & LnGDP & LnREER & LnCR & LnPR \\
\hline LnTSEX & 1.000 & & & & & & & & & & \\
\hline LnTSIM & $\begin{array}{c}-0.533^{* * *} \\
(0.002)\end{array}$ & 1.000 & & & & & & & & & \\
\hline LnTSTB & $\begin{array}{c}0.784^{* * *} \\
(0.000)\end{array}$ & $\begin{array}{c}-0.943^{* * *} \\
(0.000)\end{array}$ & 1.000 & & & & & & & & \\
\hline LnOG & $\begin{array}{c}-0.408^{* *} \\
(0.021)\end{array}$ & $\begin{array}{c}0.828^{* * * *} \\
(0.000)\end{array}$ & $\begin{array}{c}-0.767^{* * *} \\
(0.000)\end{array}$ & 1.000 & & & & & & & \\
\hline LnEG & $\begin{array}{c}-0.342 \text { * } \\
(0.056)\end{array}$ & $\begin{array}{l}0.832 * * * \\
(0.0000)\end{array}$ & $\begin{array}{c}-0.744^{* * *} \\
(0.000)\end{array}$ & $\begin{array}{c}0.975^{* * *} \\
(0.000)\end{array}$ & 1.0000 & & & & & & \\
\hline LnSG & $\begin{array}{c}-0.450 * * * \\
(0.0097)\end{array}$ & $\begin{array}{c}0.822 * * * \\
(0.000)\end{array}$ & $\begin{array}{c}-0.780 * * * \\
(0.000)\end{array}$ & $\begin{array}{c}0.996^{* * *} \\
(0.000)\end{array}$ & $\begin{array}{c}0.961^{* * *} \\
(0.000)\end{array}$ & 1.000 & & & & & \\
\hline LnPG & $\begin{array}{c}-0.343 \text { * } \\
(0.055)\end{array}$ & $\begin{array}{c}0.796^{* * * *} \\
(0.000)\end{array}$ & $\begin{array}{c}-0.719 * * * \\
(0.000)\end{array}$ & $\begin{array}{c}0.986^{* * * *} \\
(0.000)\end{array}$ & $\begin{array}{c}0.950 * * * \\
(0.000)\end{array}$ & $\begin{array}{c}0.975^{* * *} \\
(0.000)\end{array}$ & 1.000 & & & & \\
\hline LnGDP & $\begin{array}{c}-0.405^{* *} \\
(0.021)\end{array}$ & $\begin{array}{c}0.809^{* * * *} \\
(0.000)\end{array}$ & $\begin{array}{c}-0.753^{* * *} \\
(0.000)\end{array}$ & $\begin{array}{c}0.912 \text { *** } \\
(0.000)\end{array}$ & $\begin{array}{c}0.882 \text { *** } \\
(0.000)\end{array}$ & $\begin{array}{c}0.900 * * * \\
(0.000)\end{array}$ & $\begin{array}{c}0.891 \text { *** } \\
(0.000)\end{array}$ & 1.000 & & & \\
\hline LnREER & $\begin{array}{l}-0.210 \\
(0.248)\end{array}$ & $\begin{array}{c}-0.334 * \\
(0.061)\end{array}$ & $\begin{array}{c}0.163 \\
(0.373)\end{array}$ & $\begin{array}{c}-0.484 * * * \\
(0.005)\end{array}$ & $\begin{array}{c}-0.542 * * * \\
(0.001)\end{array}$ & $\begin{array}{c}-0.466^{* * *} \\
(0.007)\end{array}$ & $\begin{array}{c}-0.521^{* * *} \\
(0.002)\end{array}$ & $\begin{array}{l}-0.191 \\
(0.294)\end{array}$ & 1.000 & & \\
\hline LnCR & $\begin{array}{l}-0.197 \\
(0.280)\end{array}$ & $\begin{array}{c}0.607^{* * * *} \\
(0.000)\end{array}$ & $\begin{array}{c}-0.522 * * * \\
(0.002)\end{array}$ & $\begin{array}{c}0.747^{* * *} \\
(0.000)\end{array}$ & $\begin{array}{c}0.696^{* * *} \\
(0.000)\end{array}$ & $\begin{array}{c}0.735^{* * *} \\
(0.000)\end{array}$ & $\begin{array}{c}0.758^{* * *} \\
(0.000)\end{array}$ & $\begin{array}{c}0.657^{* * *} \\
(0.000)\end{array}$ & $\begin{array}{c}-0.328^{*} \\
(0.067)\end{array}$ & 1.000 & \\
\hline LnPR & $\begin{array}{l}0.425^{* *} \\
(0.015)\end{array}$ & $\begin{array}{l}-0.152 \\
(0.406)\end{array}$ & $\begin{array}{c}0.278 \\
(0.123)\end{array}$ & $\begin{array}{l}-0.020 \\
(0.915)\end{array}$ & $\begin{array}{l}-0.028 \\
(0.881)\end{array}$ & $\begin{array}{l}-0.040 \\
(0.829)\end{array}$ & $\begin{array}{c}0.029 \\
(0.877)\end{array}$ & $\begin{array}{l}-0.189 \\
(0.302)\end{array}$ & $\begin{array}{c}-0.322 \\
(0.072)\end{array}$ & $\begin{array}{c}0.550 * * * \\
(0.001)\end{array}$ & 1.000 \\
\hline
\end{tabular}

Notes: P-values are in parentheses. ${ }^{* * *}, * *$, and ${ }^{*}$ indicate the $1 \%, 5 \%$, and $10 \%$ significance levels, respectively. All variables are in natural logarithms. 


\section{Empirical Results}

\subsection{Unit Root Test and ARDL Bounds Tests}

In time-series analysis, the variables must be tested for stationarity before estimating the relationship between the variables. For this purpose, we adopted the ADF tests [59] and the Phillips-Perron test [60]. The results in Table 3 show that some variables are stationary in levels, I(0), while others are stationary in differences, I(1). This suggests that traditional cointegration technology, i.e., Johonsen's cointegration approach [61] which asks that all variables should be I(1), would not be suitable. However, the ARDL approach does not require prior information on the time-series variables' orders of integration, i.e., irrespective of I(0) or I(1). Thus, we use the ARDL model herein. According to the results of the bounds tests in Table 4, all F-statistics are greater than the critical value of I(1) at the $10 \%$ significant level. Therefore, the conclusion is reached that a cointegrating relationship exists between the examined variables.

Table 3. ADF and PP unit root tests for variables.

\begin{tabular}{|c|c|c|c|c|}
\hline \multirow[b]{2}{*}{ Variables } & \multicolumn{2}{|c|}{ ADF Test } & \multicolumn{2}{|c|}{ PP Test } \\
\hline & $\begin{array}{l}\text { Model with } \\
\text { Constant }\end{array}$ & $\begin{array}{c}\text { Model with Constant } \\
\text { and Trend }\end{array}$ & $\begin{array}{l}\text { Model with } \\
\text { Constant }\end{array}$ & $\begin{array}{l}\text { Model with Constant } \\
\text { and Trend }\end{array}$ \\
\hline \multicolumn{5}{|c|}{ Variables in level } \\
\hline LnTSEX & $-2.11(0)$ & $-2.47(0)$ & -2.21 & -2.47 \\
\hline LnTSIM & $-0.95(3)$ & $-1.92(3)$ & $-2.75 *$ & $-3.90 * *$ \\
\hline LnTSTB & $-1.87(0)$ & $-2.48(1)$ & -2.00 & -2.70 \\
\hline LnOG & $-2.47(0)$ & $-0.89(0)$ & $-3.26 * *$ & -0.44 \\
\hline LnEG & $-3.62 * *(3)$ & $-1.79(3)$ & $-5.66^{* * *}$ & -2.76 \\
\hline LnSG & $-2.07(0)$ & $-1.03(0)$ & -2.49 & -0.77 \\
\hline LnPG & $-1.88(0)$ & $-1.25(0)$ & -2.07 & -1.23 \\
\hline LnCR & $-1.82(0)$ & $-2.50(0)$ & -1.76 & -2.56 \\
\hline LnPR & $-2.22(0)$ & $-2.25(0)$ & -2.29 & -2.31 \\
\hline LnGDP & $0.38(4)$ & $-4.69^{* * *}(3)$ & -0.05 & -1.63 \\
\hline LnREER & $-3.46^{* *}(0)$ & $-2.86(0)$ & $-3.41^{* *}$ & $-3.98^{* *}$ \\
\hline \multicolumn{5}{|c|}{ Variables in difference } \\
\hline DLnTSEX & $-5.46^{* * *}(0)$ & $-5.40^{* * *}(0)$ & $-5.48 * * *$ & $-5.41 * * *$ \\
\hline DLnTSIM & $-5.10^{* * *}(2)$ & $-4.96^{* * *}(2)$ & $-9.72 * * *$ & $-8.90 * * *$ \\
\hline DLnTSTB & $-5.07^{* * *}(0)$ & $-4.97^{* * *}(0)$ & $-5.34 * * *$ & $-5.23 * * *$ \\
\hline DLnOG & $-4.92^{* * *}(0)$ & $-6.20^{* * *}(4)$ & $-4.92 * * *$ & $-9.12 * * *$ \\
\hline DLnEG & $-4.57^{* * *}(1)$ & $-5.36^{* * *}(2)$ & $-4.74 * * *$ & $-6.76^{* * *}$ \\
\hline DLnSG & $-5.26^{* * *}(0)$ & $-4.72^{* * *}(4)$ & $-5.26 * * *$ & $-7.64 * * *$ \\
\hline DLnPG & $-4.83^{* * *}(0)$ & $-5.55^{* * *}(4)$ & $-4.82 * * *$ & $-5.92 * * *$ \\
\hline DLnCR & $-4.12^{* * *}(2)$ & $-4.14^{* *}(2)$ & $-8.10 * * *$ & $-9.00^{* * *}$ \\
\hline DLnPR & $-5.74^{* * *}(0)$ & $-4.14^{* *}(2)$ & $-6.47^{* * *}$ & $-7.22 * * *$ \\
\hline DLnGDP & $-3.58^{* *}(1)$ & $-3.45 *(1)$ & $-2.92 *$ & -2.88 \\
\hline DLnREER & $-4.30^{* * *}(0)$ & $-5.04^{* * *}(0)$ & $-4.22 * * *$ & $-7.90^{* * *}$ \\
\hline
\end{tabular}

Note: The lags of models are in parentheses. ${ }^{* * *}, * *$, and $*$ indicate the $1 \%, 5 \%$, and $10 \%$ significance levels, respectively.

\subsection{Estimation of the ARDL Models}

Table 5 presents the estimated results of tourism service export models for the long run. The results show a long-run negative relationship between globalization (overall index as well as sub-indices) and tourism service exports. The coefficients of political risks are positive, indicating that a more stable political environment could facilitate the freedom of tourism trade between China and other countries [13] and provide a safe travel condition for foreign tourists, which could further promote tourism service exports. Except for the model with economic globalization, the positive coefficients of interaction effects indicate that stable environments create conditions to mitigate the adverse impacts of globalization.

As for control variables, GDP has a positive impact on tourism service exports, which is consistent with previous findings [62,63]. Moreover, higher real effective exchange rates lead to fewer tourism service exports [64]. 
Tables 6 and 7 show the short-run results for tourism service exports. We note that the sum of the coefficients of globalization is positive. Except for political globalization, the sum of the coefficients of the interaction term is negative, indicating that a stable environment could mitigate the positive impacts of globalization. The coefficients of the error-correction term are significantly negative at the $1 \%$ level, which confirms the long-run relationship between the variables. For all equations, the longest time for the deviation to return to the long-run equilibrium is one year and the shortest time is about four months, implying that the speeds of adjustment to equilibrium after a shock are quite fast.

Table 8 presents the estimated results of the tourism service imports models for the long run, for which globalization has significantly positive effects on tourism service imports. The coefficients of country risks are significantly negative and the positive effects of globalization even diminish with country stability. These indicate that as country risks decrease, China's tourism service imports will decline. The reason for this may be that a more stable government and environment could encourage investments in the tourism sector [65], improving the quality of tourism services and products to attract more domestic tourists, and thus decrease tourism service imports. 
Table 4. Autoregressive distributed lag (ARDL) bound test.

\begin{tabular}{|c|c|c|c|c|}
\hline \multirow{2}{*}{ Estimated Model } & \multicolumn{4}{|c|}{ F-Statistic } \\
\hline & OG & EG & SG & PG \\
\hline $\operatorname{LnTSEX}_{\mathrm{t}}=\mathrm{f}\left(\mathrm{LnGLOB}_{\mathrm{t}}, \mathrm{LnCR}_{\mathrm{t}}, \mathrm{LnGDP}_{\mathrm{t}}, \mathrm{LnREER}_{\mathrm{t}}\right)$ & $4.80 * * *$ & $7.96^{* * *}$ & $3.97 * *$ & $7.52 * * *$ \\
\hline $\operatorname{LnTSEX}_{\mathrm{t}}=\mathrm{f}\left(\mathrm{LnGLOB}_{\mathrm{t}}, \mathrm{LnPR}_{\mathrm{t}}, \mathrm{LnGDP}_{\mathrm{t}}, \mathrm{LnREER}_{\mathrm{t}}\right)$ & $6.37^{* * *}$ & $7.68^{* * *}$ & $3.78 * *$ & $4.66^{* * *}$ \\
\hline LnTSIM $_{t}=f\left(\right.$ LnGLOB $_{t}$, LnCR $_{t}$, LnGDP $_{t}$, LnREER $\left._{t}\right)$ & $12.69^{* * *}$ & $12.98^{* * *}$ & $6.46^{* * *}$ & $4.35 * *$ \\
\hline $\operatorname{LnTSIM}_{\mathrm{t}}=\mathrm{f}\left(\mathrm{LnGLOB}_{\mathrm{t}}, \mathrm{LnPR}_{\mathrm{t}}, \mathrm{LnGDP}_{\mathrm{t}}, \mathrm{LnREER}_{\mathrm{t}}\right)$ & $5.63^{* * *}$ & $16.23^{* * *}$ & $10.08^{* * *}$ & $7.12 * * *$ \\
\hline LnTSTB $_{\mathrm{t}}=\mathrm{f}\left(\mathrm{LnGLOB}_{\mathrm{t}}, \mathrm{LnCR}_{\mathrm{t}}, \mathrm{LnGDP}_{\mathrm{t}}, \mathrm{LnREER}_{\mathrm{t}}\right)$ & $3.81 * *$ & $4.49^{* * *}$ & $3.88 * *$ & $6.89 * * *$ \\
\hline $\operatorname{LnTSTB}_{\mathrm{t}}=\mathrm{f}\left(\mathrm{LnGLOB}_{\mathrm{t}}, \mathrm{LnPR}_{\mathrm{t}}, \mathrm{LnGDP}_{\mathrm{t}}, \mathrm{LnREER}_{\mathrm{t}}\right)$ & $4.58^{* * *}$ & $7.69^{* * *}$ & $3.69 * *$ & $4.49^{* * *}$ \\
\hline $\operatorname{LnTSEX}_{\mathrm{t}}=\mathrm{f}\left(\mathrm{LnGLOB} \mathrm{LnGLOB}_{\mathrm{t}}{ }^{*} \operatorname{LnCR}_{\mathrm{t}}, \mathrm{LnGDP}_{\mathrm{t}}, \mathrm{LnREER}_{\mathrm{t}}\right)$ & $3.90 * *$ & $9.17^{* * *}$ & $23.36^{* * *}$ & $3.68^{* *}$ \\
\hline $\operatorname{LnTSEX}_{\mathrm{t}}=\mathrm{f}\left(\mathrm{LnGLOB} \mathrm{LnGLOB}_{\mathrm{t}}{ }^{*} \operatorname{LnPR}_{\mathrm{t}}, \mathrm{LnGDP}_{\mathrm{t}}, \mathrm{LnREER}_{\mathrm{t}}\right)$ & $5.02 * * *$ & $3.35^{* *}$ & $4.77^{* * *}$ & $4.55^{* * *}$ \\
\hline $\operatorname{LnTSIM}_{\mathrm{t}}=\mathrm{f}\left(\mathrm{LnGLOB}_{\mathrm{t}}, \mathrm{LnGLOB}_{\mathrm{t}}^{*} \operatorname{LnCR}_{\mathrm{t}}, \mathrm{LnGDP}_{\mathrm{t}}, \mathrm{LnREER}_{\mathrm{t}}\right)$ & $12.82 * * *$ & $13.41^{* * *}$ & $8.19^{* * *}$ & $5.25^{* * *}$ \\
\hline $\operatorname{LnTSIM}_{\mathrm{t}}=\mathrm{f}\left(\mathrm{LnGLOB}_{\mathrm{t}}, \mathrm{LnGLOB}_{\mathrm{t}}{ }^{*} \operatorname{LnPR}_{\mathrm{t}}, \mathrm{LnGDP}_{\mathrm{t}}\right.$, LnREER $\left._{\mathrm{t}}\right)$ & $15.69^{* * *}$ & $4.01^{* *}$ & $11.81^{* * *}$ & $7.59 * * *$ \\
\hline $\operatorname{LnTSTB}_{\mathrm{t}}=\mathrm{f}\left(\mathrm{LnGLOB}_{\mathrm{t}}, \mathrm{LnGLOB}_{\mathrm{t}}{ }^{*} \operatorname{LnCR}_{\mathrm{t}}, \mathrm{LnGDP}_{\mathrm{t}}, \mathrm{LnREER}_{\mathrm{t}}\right)$ & $4.16^{* *}$ & $6.67^{* * *}$ & $4.04^{* *}$ & $7.67^{* * *}$ \\
\hline $\operatorname{LnTSTB}_{\mathrm{t}}=\mathrm{f}\left(\mathrm{LnGLOB}_{\mathrm{t}}, \mathrm{LnGLOB}_{\mathrm{t}}^{*} \operatorname{LnPR}_{\mathrm{t}}, \mathrm{LnGDP}_{\mathrm{t}}, \mathrm{LnREER}_{\mathrm{t}}\right)$ & $3.39 *$ & $7.19^{* * *}$ & $3.64^{* *}$ & $6.29 * * *$ \\
\hline \multicolumn{5}{|c|}{ Critical value } \\
\hline $10 \%$ & \multicolumn{2}{|c|}{$5 \%$} & \multicolumn{2}{|c|}{$1 \%$} \\
\hline $\mathrm{I}(0)$ & $\mathrm{I}(0)$ & $\mathrm{I}(1)$ & $\mathrm{I}(0)$ & $\mathrm{I}(1)$ \\
\hline 2.20 & 2.56 & 3.49 & 3.29 & 4.37 \\
\hline
\end{tabular}

Note: ${ }^{* * *}, * *$ and $*$ indicate the $1 \%, 5 \%$, and $10 \%$ significance levels, respectively. 
Table 5. The long-run estimated results for tourism service exports.

\begin{tabular}{|c|c|c|c|c|c|c|c|c|}
\hline \multirow{2}{*}{$\begin{array}{c}\begin{array}{c}\text { Globalization } \\
\text { Variables }\end{array} \\
\text { LnGLOB }\end{array}$} & \multicolumn{2}{|c|}{ Overall Globalization } & \multicolumn{2}{|c|}{ Economic Globalization } & \multicolumn{2}{|c|}{ Social Globalization } & \multicolumn{2}{|c|}{ Political Globalization } \\
\hline & $\begin{array}{c}-1.95^{* * *} \\
(0.58)\end{array}$ & $\begin{array}{c}-8.04^{* *} \\
(2.29)\end{array}$ & $\begin{array}{l}-0.95 \\
(0.93)\end{array}$ & $\begin{array}{l}2.97^{* *} \\
(1.32)\end{array}$ & $\begin{array}{c}-0.89^{* * *} \\
(0.26)\end{array}$ & \multirow[t]{2}{*}{$\begin{array}{c}-5.90 * * * \\
(0.78)\end{array}$} & \multirow{2}{*}{$\begin{array}{c}-2.60 * * \\
(0.96) \\
-3.10^{* * *} \\
(0.87)\end{array}$} & \multirow[t]{2}{*}{$\begin{array}{l}-5.15^{*} \\
(2.14)\end{array}$} \\
\hline LnCR & $\begin{array}{l}-0.23 \\
(0.97)\end{array}$ & & $\begin{array}{c}-3.29^{* * * *} \\
(0.95)\end{array}$ & & $\begin{array}{l}-0.25 \\
(1.20)\end{array}$ & & & \\
\hline LnGLOB*LnCR & & $\begin{array}{l}0.92 \text { * } \\
(0.37)\end{array}$ & & $\begin{array}{c}-0.89 * * * \\
(0.20)\end{array}$ & & $\begin{array}{c}1.04^{* * *} \\
(0.16)\end{array}$ & & $\begin{array}{c}0.06 \\
(0.31)\end{array}$ \\
\hline LnGDP & $\begin{array}{c}0.58^{* * *} \\
(0.16)\end{array}$ & $\begin{array}{c}0.98^{* * *} \\
(0.18)\end{array}$ & $\begin{array}{c}0.13 \\
(0.14)\end{array}$ & $\begin{array}{c}0.10 \\
(0.12)\end{array}$ & $\begin{array}{c}0.56^{* * *} \\
(0.13)\end{array}$ & $\begin{array}{c}0.63^{* * *} \\
(0.04)\end{array}$ & $\begin{array}{c}0.91^{* * *} \\
(0.29)\end{array}$ & $\begin{array}{l}1.32 * * \\
(0.29)\end{array}$ \\
\hline LnREER & $\begin{array}{c}-1.40^{* *} \\
(0.49)\end{array}$ & $\begin{array}{c}-1.82 \text { ** } \\
(0.50)\end{array}$ & $\begin{array}{c}-0.83 \text { * } \\
(0.46)\end{array}$ & $\begin{array}{l}-0.72 \text { * } \\
(0.34)\end{array}$ & $\begin{array}{c}-2.13^{* * *} \\
(0.40)\end{array}$ & $\begin{array}{c}-1.05^{* * *} \\
(0.16)\end{array}$ & $\begin{array}{c}-2.73^{* * *} \\
(0.87)\end{array}$ & $\begin{array}{c}-3.81^{* *} \\
(0.89)\end{array}$ \\
\hline \multirow[t]{2}{*}{ Constant } & $\begin{array}{c}13.37^{* * * *} \\
(3.53)\end{array}$ & $\begin{array}{c}20.13^{* * * *} \\
(3.70)\end{array}$ & $\begin{array}{c}22.71 * * * \\
(3.61)\end{array}$ & $\begin{array}{l}7.91 * * \\
(3.12)\end{array}$ & $\begin{array}{c}13.08^{* *} \\
(5.52)\end{array}$ & $\begin{array}{c}8.33^{* * * *} \\
(0.76)\end{array}$ & $\begin{array}{c}32.72 * * * \\
(6.01)\end{array}$ & $\begin{array}{c}31.55^{* * *} \\
(6.40)\end{array}$ \\
\hline & & & \multicolumn{3}{|c|}{ Panel B: Consider political risk in the models } & & & \\
\hline LnGLOB & $\begin{array}{c}-2.41^{* * *} \\
(0.31)\end{array}$ & $\begin{array}{c}-5.23 * * * \\
(1.10)\end{array}$ & $\begin{array}{c}-3.76^{* * *} \\
(1.02)\end{array}$ & $\begin{array}{c}0.36 \\
(1.21)\end{array}$ & $\begin{array}{l}-1.01 \\
(0.55)\end{array}$ & $\begin{array}{c}-4.75^{* *} \\
(1.31)\end{array}$ & $\begin{array}{c}-4.82^{* * *} \\
(0.86)\end{array}$ & $\begin{array}{c}-5.12^{* * *} \\
(1.24)\end{array}$ \\
\hline LnPR & $\begin{array}{c}0.69 \\
(0.61)\end{array}$ & & $\begin{array}{c}2.25 \\
(1.51)\end{array}$ & & $\begin{array}{c}3.04 \\
(2.17)\end{array}$ & & $\begin{array}{l}-1.86 \\
(1.23)\end{array}$ & \\
\hline 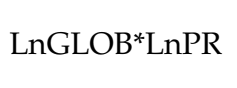 & & $\begin{array}{l}0.54^{* *} \\
(0.21)\end{array}$ & & $\begin{array}{c}-0.61 \text { ** } \\
(0.25)\end{array}$ & & $\begin{array}{l}0.82 * * \\
(0.28)\end{array}$ & & $\begin{array}{c}0.29 \\
(0.22)\end{array}$ \\
\hline LnGDP & $\begin{array}{c}0.74^{* * *} \\
(0.11)\end{array}$ & $\begin{array}{c}0.86^{* * *} \\
(0.13)\end{array}$ & $\begin{array}{c}0.44 \\
(0.25)\end{array}$ & $\begin{array}{c}0.09 \\
(0.16)\end{array}$ & $\begin{array}{c}0.28 \\
(0.50)\end{array}$ & $\begin{array}{c}0.68^{* * * *} \\
(0.13)\end{array}$ & $\begin{array}{c}1.29^{* * *} \\
(0.27)\end{array}$ & $\begin{array}{c}1.09 * * * \\
(0.27)\end{array}$ \\
\hline LnREER & $\begin{array}{c}-1.64^{* * *} \\
(0.34)\end{array}$ & $\begin{array}{c}-1.25^{* *} \\
(0.45)\end{array}$ & $\begin{array}{l}-0.29 \\
(0.50)\end{array}$ & $\begin{array}{c}-1.25^{* *} \\
(0.53)\end{array}$ & $\begin{array}{c}0.77 \\
(1.89)\end{array}$ & $\begin{array}{c}-1.06^{* *} \\
(0.38)\end{array}$ & $\begin{array}{c}-4.34^{* * *} \\
(0.89)\end{array}$ & $\begin{array}{c}-2.62^{* * *} \\
(0.66)\end{array}$ \\
\hline Constant & $\begin{array}{c}11.36^{* * *} \\
(3.22)\end{array}$ & $\begin{array}{c}13.95^{* * * *} \\
(2.48)\end{array}$ & $\begin{array}{c}5.72 \\
(4.70)\end{array}$ & $\begin{array}{c}15.68^{* * *} \\
(4.05)\end{array}$ & $\begin{array}{l}-11.58 \\
(10.13)\end{array}$ & $\begin{array}{c}7.97^{* * * *} \\
(1.61)\end{array}$ & $\begin{array}{c}41.28^{* * *} \\
(8.80)\end{array}$ & $\begin{array}{c}23.61^{* * *} \\
(4.46)\end{array}$ \\
\hline
\end{tabular}


Table 6. The estimated results of the error-correction model for tourism service exports by the ARDL model when considering the composite risk.

\begin{tabular}{|c|c|c|c|c|c|c|c|c|}
\hline \multirow[b]{2}{*}{ Variables } & \multicolumn{2}{|c|}{ Overall Globalization } & \multicolumn{2}{|c|}{ Economic Globalization } & \multicolumn{2}{|c|}{ Social Globalization } & \multicolumn{2}{|c|}{ Political Globalization } \\
\hline & $\begin{array}{c}\text { ARDL } \\
(3,2,3,3,2)\end{array}$ & $\begin{array}{c}\text { ARDL } \\
(3,3,4,4,4)\end{array}$ & $\begin{array}{c}\text { ARDL } \\
(3,3,3,3,2)\end{array}$ & $\begin{array}{c}\text { ARDL } \\
(3,1,3,3,2)\end{array}$ & $\begin{array}{c}\text { ARDL } \\
(1,0,3,0,3)\end{array}$ & $\begin{array}{c}\text { ARDL } \\
(5,3,3,3,3)\end{array}$ & $\begin{array}{c}\text { ARDL } \\
(1,0,2,3,0)\end{array}$ & $\begin{array}{c}\text { ARDL } \\
(4,4,3,4,4)\end{array}$ \\
\hline $\mathrm{D}(\operatorname{LnGLOB})_{\mathrm{t}}$ & $\begin{array}{l}-0.46 \\
(0.65)\end{array}$ & $\begin{array}{c}-11.64^{* * *} \\
(1.91)\end{array}$ & $\begin{array}{l}-0.26 \\
(0.53)\end{array}$ & $\begin{array}{l}2.32 * * \\
(0.77)\end{array}$ & & $\begin{array}{c}-3.88^{* * *} \\
(0.54)\end{array}$ & & $\begin{array}{c}-5.78^{* *} \\
(1.35)\end{array}$ \\
\hline $\mathrm{D}(\operatorname{LnGLOB})_{\mathrm{t}-1}$ & $\begin{array}{l}2.95^{* * *} \\
(0.89)\end{array}$ & $\begin{array}{c}8.86^{* * *} \\
(1.93)\end{array}$ & $\begin{array}{l}0.567 \\
(0.51)\end{array}$ & & & $\begin{array}{c}3.34^{* * *} \\
(0.57)\end{array}$ & & $\begin{array}{l}8.70 * * \\
(2.18)\end{array}$ \\
\hline $\mathrm{D}(\operatorname{LnGLOB})_{\mathrm{t}-2}$ & & $\begin{array}{c}7.79 * * * \\
(1.51)\end{array}$ & $\begin{array}{c}-2.40^{* * *} \\
(0.52)\end{array}$ & & & $\begin{array}{c}4.67^{* * * *} \\
(0.43)\end{array}$ & & $\begin{array}{l}2.95 * \\
(1.24)\end{array}$ \\
\hline $\mathrm{D}(\operatorname{LnGLOB})_{\mathrm{t}-3}$ & & & & & & & & $\begin{array}{c}8.58^{* * * *} \\
(1.49)\end{array}$ \\
\hline $\mathrm{D}(\mathrm{LnCR})_{\mathrm{t}}$ & $\begin{array}{c}0.50 \\
(0.69)\end{array}$ & & $\begin{array}{c}-2.26^{* * *} \\
(0.60)\end{array}$ & & $\begin{array}{c}-1.09 * \\
(0.59)\end{array}$ & & $\begin{array}{l}-0.45 \\
(0.59)\end{array}$ & \\
\hline $\mathrm{D}(\mathrm{LnCR})_{\mathrm{t}-1}$ & $\begin{array}{l}1.38^{*} \\
(0.74)\end{array}$ & & $\begin{array}{c}0.57 \\
(0.57)\end{array}$ & & $\begin{array}{c}0.52 \\
(0.58)\end{array}$ & & $\begin{array}{c}3.88^{* * * *} \\
(0.76)\end{array}$ & \\
\hline $\mathrm{D}(\operatorname{LnCR})_{\mathrm{t}-2}$ & $\begin{array}{c}-1.67^{* *} \\
(0.70)\end{array}$ & & $\begin{array}{c}-2.17^{* * *} \\
(0.58)\end{array}$ & & $\begin{array}{c}-2.13^{* * *} \\
(0.65)\end{array}$ & & & \\
\hline $\mathrm{D}\left(\mathrm{LnGLOB}^{*} \operatorname{LnCR}\right)_{\mathrm{t}}$ & & $\begin{array}{c}1.49 * * * \\
(0.31)\end{array}$ & & $\begin{array}{c}-0.60^{* * *} \\
(0.09)\end{array}$ & & $\begin{array}{c}0.60 * * * \\
(0.12)\end{array}$ & & $\begin{array}{c}0.46 \\
(0.27)\end{array}$ \\
\hline $\mathrm{D}\left(\mathrm{LnGLOB}^{*} \operatorname{LnCR}\right)_{\mathrm{t}-1}$ & & $\begin{array}{l}-0.26 \\
(0.21)\end{array}$ & & $\begin{array}{c}0.10 \\
(0.09)\end{array}$ & & $\begin{array}{c}-0.35 \text { ** } \\
(0.11)\end{array}$ & & $\begin{array}{c}-0.67 * \\
(0.29)\end{array}$ \\
\hline $\mathrm{D}\left(\mathrm{LnGLOB}^{*} \operatorname{LnCR}\right)_{\mathrm{t}-2}$ & & $\begin{array}{c}-1.35^{* * *} \\
(0.23)\end{array}$ & & $\begin{array}{c}-0.60^{* * *} \\
(0.09)\end{array}$ & & $\begin{array}{c}-0.98^{* * *} \\
(0.08)\end{array}$ & & $\begin{array}{c}0.44 \\
(0.29)\end{array}$ \\
\hline $\mathrm{D}\left(\mathrm{LnGLOB}^{*} \mathrm{LnCR}\right)_{\mathrm{t}-3}$ & & $\begin{array}{l}0.37^{* *} \\
(0.10)\end{array}$ & & & & & & \\
\hline $\mathrm{ECT}(-1)$ & $\begin{array}{c}-1.97 * * * \\
(0.30)\end{array}$ & $\begin{array}{c}-2.92^{* * *} \\
(0.43)\end{array}$ & $\begin{array}{c}-1.45^{* * *} \\
(0.17)\end{array}$ & $\begin{array}{c}-1.41^{* * *} \\
(0.16)\end{array}$ & $\begin{array}{c}-1.00 * * * \\
(0.180)\end{array}$ & $\begin{array}{c}-2.68^{* * *} \\
(0.16)\end{array}$ & $\begin{array}{c}-0.97^{* * *} \\
(0.13)\end{array}$ & $\begin{array}{c}-2.37^{* * *} \\
(0.34)\end{array}$ \\
\hline
\end{tabular}

Notes: Standard errors are in parentheses. ${ }^{* * *}, * *$, and ${ }^{*}$ indicate that the parameters are significant at the $1 \%, 5 \%$, and $10 \%$ levels, respectively. To save space, we only report the short-run

coefficients of globalization, country risks, and ECT (-1). 
Table 7. The estimated results of the error-correction model for tourism service exports by the ARDL model when considering the political risk.

\begin{tabular}{|c|c|c|c|c|c|c|c|c|}
\hline \multirow{2}{*}{ Variables } & \multicolumn{2}{|c|}{ Overall Globalization } & \multicolumn{2}{|c|}{ Economic Globalization } & \multicolumn{2}{|c|}{ Social Globalization } & \multicolumn{2}{|c|}{ Political Globalization } \\
\hline & $\begin{array}{c}\text { ARDL } \\
(3,2,3,3,2)\end{array}$ & $\begin{array}{c}\text { ARDL } \\
(3,3,4,4,4)\end{array}$ & $\begin{array}{c}\text { ARDL } \\
(3,4,3,4,1)\end{array}$ & $\begin{array}{c}\text { ARDL } \\
(3,0,3,3,2)\end{array}$ & $\begin{array}{c}\text { ARDL } \\
(3,4,4,1,4)\end{array}$ & $\begin{array}{c}\text { ARDL } \\
(3,3,3,4,4)\end{array}$ & $\begin{array}{c}\text { ARDL } \\
(\mathbf{1}, 3,2,3,2)\end{array}$ & $\begin{array}{c}\text { ARDL } \\
(\mathbf{1}, \mathbf{1}, \mathbf{2}, \mathbf{0}, \mathbf{0})\end{array}$ \\
\hline $\mathrm{D}(\operatorname{LnGLOB})_{\mathrm{t}}$ & $\begin{array}{l}-0.85 \\
(0.56)\end{array}$ & $\begin{array}{c}-4.80^{* * *} \\
(0.83)\end{array}$ & $\begin{array}{c}-3.09^{* * *} \\
(0.64)\end{array}$ & & $\begin{array}{c}-1.68^{* * *} \\
(0.25)\end{array}$ & $\begin{array}{l}-1.93 * * \\
(0.72)\end{array}$ & $\begin{array}{c}-3.23^{* * *} \\
(0.64)\end{array}$ & $\begin{array}{c}-2.13^{* * *} \\
(0.62)\end{array}$ \\
\hline $\mathrm{D}(\operatorname{LnGLOB})_{\mathrm{t}-1}$ & $\begin{array}{l}4.19^{* * *} \\
(0.84)\end{array}$ & $\begin{array}{l}4.50^{* *} \\
(1.14)\end{array}$ & $\begin{array}{l}4.91^{* * * *} \\
(0.75)\end{array}$ & & & $\begin{array}{l}2.52 * * \\
(0.93)\end{array}$ & $\begin{array}{l}1.79 * * \\
(0.63)\end{array}$ & \\
\hline $\mathrm{D}(\operatorname{LnGLOB})_{\mathrm{t}-2}$ & & $\begin{array}{l}4.07^{* * * *} \\
(0.85)\end{array}$ & $\begin{array}{c}-2.52^{* * *} \\
(0.52)\end{array}$ & & & & $\begin{array}{l}1.80 * * \\
(0.83)\end{array}$ & \\
\hline $\mathrm{D}(\operatorname{LnGLOB})_{\mathrm{t}-3}$ & & & $\begin{array}{c}2.80 * * * \\
(0.64)\end{array}$ & & & & & \\
\hline $\mathrm{D}(\mathrm{LnPR})_{\mathrm{t}}$ & $\begin{array}{c}0.76 \\
(0.49)\end{array}$ & & $\begin{array}{l}1.12 * * \\
(0.48)\end{array}$ & & $\begin{array}{l}-0.12 \\
(0.56)\end{array}$ & & $\begin{array}{c}-2.17^{* *} \\
(0.93)\end{array}$ & \\
\hline $\mathrm{D}(\mathrm{LnPR})_{\mathrm{t}-1}$ & $\begin{array}{c}0.42 \\
(0.50)\end{array}$ & & $\begin{array}{c}-2.45^{* * *} \\
(0.60)\end{array}$ & & $\begin{array}{l}1.66^{* *} \\
(0.51)\end{array}$ & & $\begin{array}{c}2.44^{* * *} \\
(0.58)\end{array}$ & \\
\hline $\mathrm{D}(\mathrm{LnPR})_{\mathrm{t}-2}$ & $\begin{array}{c}-2.19^{* * *} \\
(0.63)\end{array}$ & & $\begin{array}{c}-1.67^{* * *} \\
(0.49)\end{array}$ & & $\begin{array}{l}-1.73^{* *} \\
(0.56)\end{array}$ & & & \\
\hline $\mathrm{D}(\mathrm{LnPR})_{\mathrm{t}-3}$ & & & & & $\begin{array}{l}1.88^{* * *} \\
(0.44)\end{array}$ & & & \\
\hline $\mathrm{D}\left(\mathrm{LnGLOB}^{*} \mathrm{LnPR}\right)_{\mathrm{t}}$ & & $\begin{array}{c}0.33 \\
(0.17)\end{array}$ & & $\begin{array}{l}-0.32 * \\
(0.15)\end{array}$ & & $\begin{array}{l}-0.05 \\
(0.16)\end{array}$ & & $\begin{array}{c}0.17 \\
(0.11)\end{array}$ \\
\hline $\mathrm{D}\left(\operatorname{LnGLOB}^{*} \operatorname{LnPR}\right)_{\mathrm{t}-1}$ & & $\begin{array}{l}-0.02 \\
(0.12)\end{array}$ & & $\begin{array}{c}0.16 \\
(0.12)\end{array}$ & & $\begin{array}{c}-0.44 \text { * } \\
(0.19)\end{array}$ & & $\begin{array}{c}0.27^{* * *} \\
(0.08)\end{array}$ \\
\hline $\mathrm{D}\left(\mathrm{LnGLOB}^{*} \mathrm{LnPR}_{\mathrm{t}-2}\right.$ & & $\begin{array}{c}-0.68^{* * *} \\
(0.14)\end{array}$ & & $\begin{array}{c}-0.47^{* * *} \\
(0.13)\end{array}$ & & $\begin{array}{c}-1.17^{* * *} \\
(0.20)\end{array}$ & & \\
\hline $\mathrm{D}\left(\mathrm{LnGLOB}^{*} \operatorname{LnPR}\right)_{\mathrm{t}-3}$ & & $\begin{array}{l}0.30 \text { ** } \\
(0.09)\end{array}$ & & & & & & \\
\hline $\mathrm{ECT}(-1)$ & $\begin{array}{c}-2.30^{* * *} \\
(0.31)\end{array}$ & $\begin{array}{c}-2.32^{* * *} \\
(0.39)\end{array}$ & $\begin{array}{c}-1.45^{* * *} \\
(0.17)\end{array}$ & $\begin{array}{c}-1.25^{* * *} \\
(0.24)\end{array}$ & $\begin{array}{c}-1.01^{* * *} \\
(0.16)\end{array}$ & $\begin{array}{c}-2.25^{* * *} \\
(0.31)\end{array}$ & $\begin{array}{c}-1.24^{* * *} \\
(0.20)\end{array}$ & $\begin{array}{c}-0.75^{* * *} \\
(0.13)\end{array}$ \\
\hline
\end{tabular}

Notes: Standard errors are in parentheses. ${ }^{* * *},{ }^{* *}$, and ${ }^{*}$ indicate that the parameters are significant at the $1 \%, 5 \%$, and $10 \%$ levels, respectively. To save space, we only report the short-run coefficients of globalization, country risks, and ECT $(-1)$. 
Table 8. The long-run estimated results for tourism service imports.

\begin{tabular}{|c|c|c|c|c|c|c|c|c|}
\hline \multirow{2}{*}{$\begin{array}{c}\begin{array}{c}\text { Globalization } \\
\text { Variables }\end{array} \\
\text { LnGLOB }\end{array}$} & \multicolumn{2}{|c|}{ Overall Globalization } & \multicolumn{2}{|c|}{ Economic Globalization } & \multicolumn{2}{|c|}{ Social Globalization } & \multicolumn{2}{|c|}{ Political Globalization } \\
\hline & & & \multicolumn{3}{|c|}{ Panel A: Consider composite risk in the models } & \multirow{3}{*}{$\begin{array}{l}5.29 * \\
(2.33)\end{array}$} & \multirow{3}{*}{$\begin{array}{c}4.17 \text { *** } \\
(0.85) \\
-1.78^{*} \\
(0.80)\end{array}$} & \multirow{3}{*}{$\begin{array}{c}6.33^{* * *} \\
(1.10)\end{array}$} \\
\hline & $\begin{array}{l}4.46^{* *} \\
(0.97)\end{array}$ & $\begin{array}{l}9.24^{* *} \\
(2.02)\end{array}$ & $\begin{array}{c}5.47^{* * *} \\
(0.93)\end{array}$ & $\begin{array}{c}11.05^{* *} \\
(1.94)\end{array}$ & $\begin{array}{l}-2.02 \\
(1.59)\end{array}$ & & & \\
\hline LnCR & $\begin{array}{c}-5.70^{* *} \\
(1.48)\end{array}$ & & $\begin{array}{c}-5.29^{* *} \\
(1.36)\end{array}$ & & $\begin{array}{l}12.10 * \\
(6.72)\end{array}$ & & & \\
\hline LnGLOB* $^{*}$ LnCR & & $\begin{array}{c}-1.27 * * \\
(0.30)\end{array}$ & & $\begin{array}{c}-1.35^{* *} \\
(0.31)\end{array}$ & & $\begin{array}{l}-0.97 \\
(0.46)\end{array}$ & & $\begin{array}{c}-0.44^{* *} \\
(0.16)\end{array}$ \\
\hline LnGDP & $\begin{array}{c}-0.60 * \\
(0.23)\end{array}$ & $\begin{array}{c}-0.47^{*} \\
(0.20)\end{array}$ & $\begin{array}{l}-0.01 \\
(0.14)\end{array}$ & $\begin{array}{c}0.02 \\
(0.13)\end{array}$ & $\begin{array}{c}1.04 \\
(0.78)\end{array}$ & $\begin{array}{l}-0.09 \\
(0.19)\end{array}$ & $\begin{array}{c}-0.71^{* *} \\
(0.24)\end{array}$ & $\begin{array}{c}-0.80^{* * *} \\
(0.18)\end{array}$ \\
\hline LnREER & $\begin{array}{c}0.98 \\
(0.51)\end{array}$ & $\begin{array}{c}0.80 \\
(0.46)\end{array}$ & $\begin{array}{l}-0.33 \\
(0.59)\end{array}$ & $\begin{array}{l}-0.46 \\
(0.52)\end{array}$ & $\begin{array}{c}0.61 \\
(1.55)\end{array}$ & $\begin{array}{c}0.28 \\
(0.55)\end{array}$ & $\begin{array}{l}1.92 \text { ** } \\
(0.70)\end{array}$ & $\begin{array}{c}2.22 * * * \\
(0.55)\end{array}$ \\
\hline Constant & $\begin{array}{l}10.62 * \\
(3.71)\end{array}$ & $\begin{array}{c}-11.21 \text { * } \\
(3.92)\end{array}$ & $\begin{array}{c}7.81 \\
(4.83)\end{array}$ & $\begin{array}{c}-13.58^{* *} \\
(4.01)\end{array}$ & $\begin{array}{l}-49.06^{*} \\
(24.34)\end{array}$ & $\begin{array}{l}-0.80 \\
(3.03)\end{array}$ & $\begin{array}{c}-9.75^{*} \\
(4.62)\end{array}$ & $\begin{array}{c}-19.25^{* * *} \\
(3.85)\end{array}$ \\
\hline \multicolumn{9}{|c|}{ Panel B: Consider political risk in the models } \\
\hline LnGLOB & $\begin{array}{l}3.38^{* *} \\
(1.24)\end{array}$ & $\begin{array}{l}4.52^{* *} \\
(1.19)\end{array}$ & $\begin{array}{l}2.21^{*} \\
(0.91)\end{array}$ & $\begin{array}{l}-2.93 \\
(2.67)\end{array}$ & $\begin{array}{c}0.66^{* * *} \\
(0.15)\end{array}$ & $\begin{array}{l}3.38^{* *} \\
(0.90)\end{array}$ & $\begin{array}{c}3.76^{* * *} \\
(0.37)\end{array}$ & $\begin{array}{c}5.82 * * * \\
(0.59)\end{array}$ \\
\hline LnPR & $\begin{array}{l}-3.30 \\
(2.27)\end{array}$ & & $\begin{array}{l}-0.81 \\
(2.45)\end{array}$ & & $\begin{array}{c}-2.21 \\
(0.92)\end{array}$ & & $\begin{array}{c}-2.22^{* * *} \\
(0.54)\end{array}$ & \\
\hline LnGLOB*LnPR & & $\begin{array}{c}-0.63 \text { ** } \\
(0.20)\end{array}$ & & $\begin{array}{c}0.45 \\
(0.45)\end{array}$ & & $\begin{array}{c}-0.65 \text { ** } \\
(0.19)\end{array}$ & & $\begin{array}{c}-0.51 \text { *** } \\
(0.12)\end{array}$ \\
\hline LnGDP & $\begin{array}{l}-0.10 \\
(0.43)\end{array}$ & $\begin{array}{l}-0.19 \\
(0.18)\end{array}$ & $\begin{array}{c}0.002 \\
(0.184)\end{array}$ & $\begin{array}{l}0.71 \text { * } \\
(0.34)\end{array}$ & $\begin{array}{c}0.04 \\
(0.11)\end{array}$ & $\begin{array}{l}0.003 \\
(0.14)\end{array}$ & $\begin{array}{c}-0.80 * * * \\
(0.11)\end{array}$ & $\begin{array}{c}-0.79 * * * * \\
(0.11)\end{array}$ \\
\hline LnREER & $\begin{array}{c}1.40 \\
(1.48)\end{array}$ & $\begin{array}{l}-0.14 \\
(0.28)\end{array}$ & $\begin{array}{c}0.55 \\
(1.14)\end{array}$ & $\begin{array}{l}-1.01 \\
(1.05)\end{array}$ & $\begin{array}{l}-0.47 \\
(0.27)\end{array}$ & $\begin{array}{c}-0.54^{*} \\
(0.23)\end{array}$ & $\begin{array}{c}1.81^{* * * *} \\
(0.38)\end{array}$ & $\begin{array}{c}1.79 * * * \\
(0.37)\end{array}$ \\
\hline Constant & $\begin{array}{c}-2.63 \\
(12.09)\end{array}$ & $\begin{array}{l}-1.59 \\
(1.80)\end{array}$ & $\begin{array}{c}-3.82 \\
(13.23)\end{array}$ & $\begin{array}{c}7.67 \\
(8.83)\end{array}$ & $\begin{array}{c}12.46^{* *} \\
(3.57)\end{array}$ & $\begin{array}{l}3.77 * * \\
(1.16)\end{array}$ & $\begin{array}{l}-5.34 \\
(3.77)\end{array}$ & $\begin{array}{c}-14.38^{* * *} \\
(2.45)\end{array}$ \\
\hline
\end{tabular}


The results present that tourism service imports are inversely associated with GDP. The reason for this may be that the travel channel has become an important route for Chinese household capital flight in recent years, even as genuine travel consumption has also increased rapidly [66]. In addition, the results illustrate that the tourism service imports increase along with RMB appreciation, which enhances the relative purchasing power and stimulates outbound tourism [67].

Tables 9 and 10 report the short-term results for tourism service imports. The sum of the coefficients of globalization is negative. The results of the sum of the coefficients of the interaction term indicate that country stability could mitigate the negative impacts of overall, economic, and social globalization on tourism service imports, while enhance the negative impacts of political globalization on tourism service imports. The error-correction term also has a negative coefficient and is highly significant for all equations. The longest time for the deviation to return to the long-run equilibrium is about two years and the shortest time is six months, implying that the speeds of adjustment to equilibrium after a shock are relatively fast.

Table 11 presents the estimated results of tourism service trade balance models for the long run. The estimated coefficients are significantly negative for globalization, indicating that globalization expands the gap between tourism service imports and exports in China. Through the aforementioned analysis, we can see that greater globalization leads to fewer tourism service exports, but more tourism service imports, and therefore, the deepening of globalization will intensify the trade deficit of China's tourism services. A more stable environment also intensifies the trade deficit of tourism services but can mitigate the effects of globalization on tourism services trade balances. Moreover, the results suggest that an increase in real GDP and a depreciation could improve the tourism service trade deficit $[67,68]$.

Tables 12 and 13 show the short-term relationships between tourism service trade balances and the determinants. The sum of the coefficients of globalization is roughly positive. The sum of the coefficients of country risks (or interaction term) is negative, which suggests that country risk indices not only have a negative impact on tourism service trade balances, but also on the relationship between globalization and tourism service trade balances. The coefficient of the error-correction term is significantly negative at the $1 \%$ level for all equations. The longest time for the deviation to return to the long-run equilibrium is more than two years and the shortest time is four months. 
Table 9. The estimated results of the error-correction model for tourism service imports by the ARDL model when considering the composite risk.

\begin{tabular}{|c|c|c|c|c|c|c|c|c|}
\hline \multirow{2}{*}{ Variables } & \multicolumn{2}{|c|}{ Overall Globalization } & \multicolumn{2}{|c|}{ Economic Globalization } & \multicolumn{2}{|c|}{ Social Globalization } & \multicolumn{2}{|c|}{ Political Globalization } \\
\hline & $\begin{array}{c}\text { ARDL } \\
(4,4,4,4,4)\end{array}$ & $\begin{array}{c}\text { ARDL } \\
(4,4,4,4,4)\end{array}$ & $\begin{array}{c}\text { ARDL } \\
(4,4,4,4,4)\end{array}$ & $\begin{array}{c}\text { ARDL } \\
(\mathbf{4}, \mathbf{4}, \mathbf{4 , 4}, \mathbf{4})\end{array}$ & $\begin{array}{c}\text { ARDL } \\
(\mathbf{1}, \mathbf{0}, \mathbf{3}, \mathbf{2}, \mathbf{0})\end{array}$ & $\begin{array}{c}\text { ARDL } \\
(4,4,4,4,3)\end{array}$ & $\begin{array}{c}\text { ARDL } \\
(3,3,3,3,3)\end{array}$ & $\begin{array}{c}\text { ARDL } \\
(2,3,3,3,3)\end{array}$ \\
\hline $\mathrm{D}(\operatorname{LnGLOB})_{\mathrm{t}}$ & $\begin{array}{l}2.02 * * \\
(0.29)\end{array}$ & $\begin{array}{l}2.80 * * \\
(0.64)\end{array}$ & $\begin{array}{c}0.38 \\
(0.26)\end{array}$ & $\begin{array}{l}2.83^{* *} \\
(0.73)\end{array}$ & & $\begin{array}{l}-1.29 \\
(0.76)\end{array}$ & $\begin{array}{c}0.52 \\
(0.57)\end{array}$ & $\begin{array}{c}-5.91 * * * \\
(1.29)\end{array}$ \\
\hline $\mathrm{D}(\operatorname{LnGLOB})_{\mathrm{t}-1}$ & $\begin{array}{c}-4.58^{* * *} \\
(0.35)\end{array}$ & $\begin{array}{c}-12.92^{* * *} \\
(0.84)\end{array}$ & $\begin{array}{c}-6.81^{* * *} \\
(0.55)\end{array}$ & $\begin{array}{c}-13.29^{* * * *} \\
(0.90)\end{array}$ & & $\begin{array}{c}-9.53^{* * *} \\
(0.88)\end{array}$ & $\begin{array}{c}-6.68^{* * *} \\
(1.28)\end{array}$ & $\begin{array}{l}-4.26^{* *} \\
(1.41)\end{array}$ \\
\hline $\mathrm{D}(\operatorname{LnGLOB})_{\mathrm{t}-2}$ & $\begin{array}{c}-2.32^{* * *} \\
(0.34)\end{array}$ & $\begin{array}{c}-9.90 * * * \\
(0.73)\end{array}$ & $\begin{array}{c}-3.97^{* * *} \\
(0.38)\end{array}$ & $\begin{array}{c}-11.47^{* * * *} \\
(0.68)\end{array}$ & & $\begin{array}{c}-8.13^{* * *} \\
(0.79)\end{array}$ & $\begin{array}{c}-3.17^{* *} \\
(1.14)\end{array}$ & \\
\hline $\mathrm{D}(\operatorname{LnGLOB})_{\mathrm{t}-3}$ & $\begin{array}{c}-2.15^{* * *} \\
(0.32)\end{array}$ & $\begin{array}{c}-5.18^{* * *} \\
(0.58)\end{array}$ & $\begin{array}{c}-3.13^{* * *} \\
(0.30)\end{array}$ & $\begin{array}{c}-8.65^{* * *} \\
(0.75)\end{array}$ & & $\begin{array}{c}-3.58^{* * *} \\
(0.63)\end{array}$ & & \\
\hline $\mathrm{D}(\mathrm{LnCR})_{\mathrm{t}}$ & $\begin{array}{l}-0.66 \\
(0.41)\end{array}$ & & $\begin{array}{l}-1.38^{*} \\
(0.47)\end{array}$ & & $\begin{array}{l}4.15^{* * *} \\
(0.63)\end{array}$ & & $\begin{array}{l}-1.05 \\
(1.15)\end{array}$ & \\
\hline $\mathrm{D}(\mathrm{LnCR})_{\mathrm{t}-1}$ & $\begin{array}{l}7.92 * * * \\
(0.52)\end{array}$ & & $\begin{array}{l}5.45^{* * *} \\
(0.38)\end{array}$ & & $\begin{array}{l}1.09 * * \\
(0.41)\end{array}$ & & $\begin{array}{l}-0.87 \\
(0.72)\end{array}$ & \\
\hline $\mathrm{D}(\mathrm{LnCR})_{\mathrm{t}-2}$ & $\begin{array}{l}7.38^{* * *} \\
(0.47)\end{array}$ & & $\begin{array}{l}6.69^{* * * *} \\
(0.46)\end{array}$ & & $\begin{array}{l}1.70^{* * *} \\
(0.46)\end{array}$ & & $\begin{array}{l}1.30 * \\
(0.64)\end{array}$ & \\
\hline $\mathrm{D}(\mathrm{LnCR})_{\mathrm{t}-3}$ & $\begin{array}{c}2.71^{* * *} \\
(0.33)\end{array}$ & & $\begin{array}{l}4.61^{* * *} \\
(0.44)\end{array}$ & & & & & \\
\hline $\mathrm{D}\left(\mathrm{LnGLOB}^{*} \operatorname{LnCR}\right)_{\mathrm{t}}$ & & $\begin{array}{l}-0.27^{*} \\
(0.11)\end{array}$ & & $\begin{array}{l}-0.53^{* *} \\
(0.13)\end{array}$ & & $\begin{array}{c}0.30 \\
(0.16)\end{array}$ & & $\begin{array}{l}-0.38 \\
(0.26)\end{array}$ \\
\hline $\mathrm{D}\left(\operatorname{LnGLOB}{ }^{*} \operatorname{LnCR}\right)_{\mathrm{t}-1}$ & & $\begin{array}{l}1.90^{* * *} \\
(0.13)\end{array}$ & & $\begin{array}{c}1.38^{* * *} \\
(0.10)\end{array}$ & & $\begin{array}{l}1.87^{* * *} \\
(0.18)\end{array}$ & & $\begin{array}{l}-0.31 \text { * } \\
(0.16)\end{array}$ \\
\hline $\mathrm{D}\left(\mathrm{LnGLOB}^{*} \operatorname{LnCR}\right)_{\mathrm{t}-2}$ & & $\begin{array}{l}1.79 * * * \\
(0.12)\end{array}$ & & $\begin{array}{c}1.72 * * * \\
(0.12)\end{array}$ & & $\begin{array}{l}1.77^{* * *} \\
(0.17)\end{array}$ & & $\begin{array}{l}0.29 * \\
(0.14)\end{array}$ \\
\hline $\mathrm{D}\left(\mathrm{LnGLOB}^{*} \operatorname{LnCR}\right)_{\mathrm{t}-3}$ & & $\begin{array}{c}0.74^{* * *} \\
(0.09)\end{array}$ & & $\begin{array}{c}1.27^{* * *} \\
(0.12)\end{array}$ & & $\begin{array}{l}0.77^{* * *} \\
(0.14)\end{array}$ & & \\
\hline $\operatorname{ECT}(-1)$ & $\begin{array}{c}-1.63^{* * *} \\
(0.11)\end{array}$ & $\begin{array}{l}-1.84^{* * *} \\
(0.13)\end{array}$ & $\begin{array}{c}-1.38^{* * *} \\
(0.10)\end{array}$ & $\begin{array}{c}-1.54^{* * * *} \\
(0.11)\end{array}$ & $\begin{array}{c}-0.28^{* * *} \\
(0.04)\end{array}$ & $\begin{array}{c}-1.57^{* * *} \\
(0.15)\end{array}$ & $\begin{array}{c}-1.76^{* * *} \\
(0.28)\end{array}$ & $\begin{array}{c}-1.80^{* * *} \\
(0.26)\end{array}$ \\
\hline
\end{tabular}

Notes: Standard errors are in parentheses. ${ }^{* * *},{ }^{* *}$, and ${ }^{*}$ indicate that the parameters are significant at the $1 \%, 5 \%$, and $10 \%$ levels, respectively. To save space, we only report the short-run coefficients of globalization, country risks, and ECT $(-1)$. 
Table 10. The estimated results of the error-correction model for tourism service imports by the ARDL model when considering the political risk.

\begin{tabular}{|c|c|c|c|c|c|c|c|c|}
\hline \multirow{2}{*}{ Variables } & \multicolumn{2}{|c|}{ Overall Globalization } & \multicolumn{2}{|c|}{ Economic Globalization } & \multicolumn{2}{|c|}{ Social Globalization } & \multicolumn{2}{|c|}{ Political Globalization } \\
\hline & $\begin{array}{c}\text { ARDL } \\
(3,3,4,4,4)\end{array}$ & $\begin{array}{c}\text { ARDL } \\
(4,4,4,4,3)\end{array}$ & $\begin{array}{c}\text { ARDL } \\
(4,4,4,4,3)\end{array}$ & $\begin{array}{c}\text { ARDL } \\
(3,0,2,2,2)\end{array}$ & $\begin{array}{c}\text { ARDL } \\
(4,3,4,4,3)\end{array}$ & $\begin{array}{c}\text { ARDL } \\
(4,4,4,4,3)\end{array}$ & $\begin{array}{c}\text { ARDL } \\
(1,3,3,3,2)\end{array}$ & $\begin{array}{c}\text { ARDL } \\
(1,3,3,3,2)\end{array}$ \\
\hline $\mathrm{D}(\operatorname{LnGLOB})_{\mathrm{t}}$ & $\begin{array}{l}2.82^{* * *} \\
(0.51)\end{array}$ & $\begin{array}{l}1.64^{* *} \\
(0.44)\end{array}$ & $\begin{array}{l}0.68^{* *} \\
(0.23)\end{array}$ & & $\begin{array}{l}0.36^{* *} \\
(0.12)\end{array}$ & $\begin{array}{c}0.27 \\
(0.48)\end{array}$ & $\begin{array}{l}-0.42 \\
(0.43)\end{array}$ & $\begin{array}{c}2.29 * * * \\
(0.65)\end{array}$ \\
\hline $\mathrm{D}(\operatorname{LnGLOB})_{\mathrm{t}-1}$ & $\begin{array}{l}2.04^{* * *} \\
(0.42)\end{array}$ & $\begin{array}{c}-7.18^{* * *} \\
(0.49)\end{array}$ & $\begin{array}{c}0.32 \\
(0.25)\end{array}$ & & $\begin{array}{l}-0.36^{* *} \\
(0.11)\end{array}$ & $\begin{array}{c}-6.02 * * * \\
(0.52)\end{array}$ & $\begin{array}{l}-7.60^{* * *} \\
(1.11)\end{array}$ & $\begin{array}{c}-7.44^{* * *} \\
(1.12)\end{array}$ \\
\hline $\mathrm{D}(\operatorname{LnGLOB})_{\mathrm{t}-2}$ & $\begin{array}{l}2.19^{* * *} \\
(0.50)\end{array}$ & $\begin{array}{c}-5.54^{* * *} \\
(0.53)\end{array}$ & $\begin{array}{c}-1.93^{* * *} \\
(0.27)\end{array}$ & & $\begin{array}{c}0.28^{*} \\
(0.11)\end{array}$ & $\begin{array}{c}-5.94^{* * *} \\
(0.57)\end{array}$ & $\begin{array}{c}-1.80^{* *} \\
(0.74)\end{array}$ & $\begin{array}{c}-3.44^{* * *} \\
(1.02)\end{array}$ \\
\hline $\mathrm{D}(\operatorname{LnGLOB})_{\mathrm{t}-3}$ & & $\begin{array}{c}-3.64^{* * *} \\
(0.39)\end{array}$ & $\begin{array}{c}-1.16^{* *} \\
(0.26)\end{array}$ & & & & & \\
\hline $\mathrm{D}(\mathrm{LnPR})_{\mathrm{t}}$ & $\begin{array}{l}3.01^{* * *} \\
(0.64)\end{array}$ & & $\begin{array}{c}3.95 * * * \\
(0.30)\end{array}$ & & $\begin{array}{c}0.63 \\
(0.38)\end{array}$ & & $\begin{array}{c}-2.87^{* * *} \\
(0.54)\end{array}$ & \\
\hline $\mathrm{D}(\mathrm{LnPR})_{\mathrm{t}-1}$ & $\begin{array}{l}5.34^{* * *} \\
(0.60)\end{array}$ & & $\begin{array}{l}5.35 * * * \\
(0.37)\end{array}$ & & $\begin{array}{l}4.33^{* * *} \\
(0.41)\end{array}$ & & $\begin{array}{c}0.03 \\
(0.38)\end{array}$ & \\
\hline $\mathrm{D}(\mathrm{LnPR})_{\mathrm{t}-2}$ & $\begin{array}{l}3.94^{* * *} \\
(0.49)\end{array}$ & & $\begin{array}{l}4.45^{* * *} \\
(0.35)\end{array}$ & & $\begin{array}{l}5.11^{* * *} \\
(0.51)\end{array}$ & & $\begin{array}{l}1.88^{* * *} \\
(0.51)\end{array}$ & \\
\hline $\mathrm{D}(\mathrm{LnPR})_{\mathrm{t}-3}$ & $\begin{array}{l}1.13^{* *} \\
(0.36)\end{array}$ & & $\begin{array}{l}1.28^{* * *} \\
(0.20)\end{array}$ & & $\begin{array}{l}2.51^{* * *} \\
(0.34)\end{array}$ & & & \\
\hline $\mathrm{D}\left(\operatorname{LnGLOB}{ }^{*} \operatorname{LnPR}\right)_{\mathrm{t}}$ & & $\begin{array}{l}-0.08 \\
(0.09)\end{array}$ & & $\begin{array}{c}0.50^{* * *} \\
(0.12)\end{array}$ & & $\begin{array}{c}0.01 \\
(0.10)\end{array}$ & & $\begin{array}{c}-0.66^{* * *} \\
(0.20)\end{array}$ \\
\hline $\mathrm{D}\left(\mathrm{LnGLOB}^{*} \operatorname{LnPR}\right)_{\mathrm{t}-1}$ & & $\begin{array}{l}1.28^{* * *} \\
(0.10)\end{array}$ & & $\begin{array}{l}0.19^{*} \\
(0.10)\end{array}$ & & $\begin{array}{l}1.27^{* * *} \\
(0.11)\end{array}$ & & $\begin{array}{l}0.001 \\
(0.09)\end{array}$ \\
\hline $\mathrm{D}\left(\mathrm{LnGLOB}^{*} \operatorname{LnPR}\right)_{\mathrm{t}-2}$ & & $\begin{array}{l}1.32^{* * *} \\
(0.10)\end{array}$ & & & & $\begin{array}{l}1.44^{* * *} \\
(0.13)\end{array}$ & & $\begin{array}{c}0.41^{* * *} \\
(0.11)\end{array}$ \\
\hline $\mathrm{D}\left(\mathrm{LnGLOB}^{*} \operatorname{LnPR}\right)_{\mathrm{t}-3}$ & & $\begin{array}{l}0.59^{* * *} \\
(0.07)\end{array}$ & & & & $\begin{array}{l}0.73^{* * *} \\
(0.09)\end{array}$ & & \\
\hline $\operatorname{ECT}(-1)$ & $\begin{array}{c}-0.62 * * * \\
(0.08)\end{array}$ & $\begin{array}{c}-1.97^{* * *} \\
(0.14)\end{array}$ & $\begin{array}{c}-1.40^{* * *} \\
(0.09)\end{array}$ & $\begin{array}{c}-0.59 * * * \\
(0.10)\end{array}$ & $\begin{array}{c}-1.63^{* * *} \\
(0.15)\end{array}$ & $\begin{array}{c}-1.99 * * * \\
(0.16)\end{array}$ & $\begin{array}{c}-1.88^{* * *} \\
(0.24)\end{array}$ & $\begin{array}{c}-1.85^{* * * *} \\
(0.23)\end{array}$ \\
\hline
\end{tabular}

Notes: Standard errors are in parentheses. ${ }^{* * *},{ }^{* *}$, and ${ }^{*}$ indicate that the parameters are significant at the $1 \%, 5 \%$, and $10 \%$ levels, respectively. To save space, we only report the short-run coefficients of globalization, country risks, and ECT $(-1)$. 
Table 11. The long-run estimated results for tourism service trade balance.

\begin{tabular}{|c|c|c|c|c|c|c|c|c|}
\hline $\begin{array}{c}\text { Globalization } \\
\text { Variables }\end{array}$ & \multicolumn{2}{|c|}{ Overall Globalization } & \multicolumn{2}{|c|}{ Economic Globalization } & \multicolumn{2}{|c|}{ Social Globalization } & \multicolumn{2}{|c|}{ Political Globalization } \\
\hline LnGLOB & $\begin{array}{l}-0.01 \\
(2.04)\end{array}$ & $\begin{array}{c}-26.25^{* * *} \\
(5.93)\end{array}$ & $\begin{array}{c}8.16 \\
(5.08)\end{array}$ & $\begin{array}{c}19.07^{* *} \\
(8.04)\end{array}$ & $\begin{array}{c}2.93 \\
(2.11)\end{array}$ & $\begin{array}{c}-13.76^{*} \\
(5.74)\end{array}$ & $\begin{array}{c}-8.05^{* * *} \\
(1.75)\end{array}$ & $\begin{array}{c}-6.54^{* *} \\
(2.25)\end{array}$ \\
\hline LnCR & $\begin{array}{l}-3.23 \\
(3.59)\end{array}$ & & $\begin{array}{c}-14.09^{* * * *} \\
(4.43)\end{array}$ & & $\begin{array}{l}-16.75^{* *} \\
(7.77)\end{array}$ & & $\begin{array}{l}-1.33 \\
(1.82)\end{array}$ & \\
\hline LnGLOB*LnCR & & $\begin{array}{l}3.50 \text { ** } \\
(0.90)\end{array}$ & & $\begin{array}{c}-2.96^{* *} \\
(1.01)\end{array}$ & & $\begin{array}{l}2.50 \text { * } \\
(1.14)\end{array}$ & & $\begin{array}{l}-0.15 \\
(0.30)\end{array}$ \\
\hline LnGDP & $\begin{array}{l}-0.39 \\
(0.57)\end{array}$ & $\begin{array}{l}1.98^{* *} \\
(0.47)\end{array}$ & $\begin{array}{l}-1.08 \\
(0.69)\end{array}$ & $\begin{array}{c}-1.72^{* *} \\
(0.79)\end{array}$ & $\begin{array}{l}-1.50 \\
(1.10)\end{array}$ & $\begin{array}{c}0.68 \\
(0.42)\end{array}$ & $\begin{array}{l}1.78^{* *} \\
(0.47)\end{array}$ & $\begin{array}{c}1.62^{* * * *} \\
(0.42)\end{array}$ \\
\hline LnREER & $\begin{array}{c}0.77 \\
(1.93)\end{array}$ & $\begin{array}{c}-2.59^{* *} \\
(0.99)\end{array}$ & $\begin{array}{c}0.36 \\
(2.03)\end{array}$ & $\begin{array}{c}2.70 \\
(1.78)\end{array}$ & $\begin{array}{l}1.88 \\
(2.76)\end{array}$ & $\begin{array}{l}-1.02 \\
(1.30)\end{array}$ & $\begin{array}{c}-5.63^{* *} \\
(1.44)\end{array}$ & $\begin{array}{c}-4.98^{* * *} \\
(1.31)\end{array}$ \\
\hline Constant & $\begin{array}{c}11.30 \\
(14.94)\end{array}$ & $\begin{array}{c}41.22 * * * \\
(9.19)\end{array}$ & $\begin{array}{l}32.87 * \\
(16.13)\end{array}$ & $\begin{array}{l}-27.26 \\
(17.38)\end{array}$ & $\begin{array}{l}58.98^{* *} \\
(23.39)\end{array}$ & $\begin{array}{l}10.14 \\
(6.77)\end{array}$ & $\begin{array}{c}51.21^{* * *} \\
(8.82)\end{array}$ & $\begin{array}{c}40.05^{* * *} \\
(8.91)\end{array}$ \\
\hline \multicolumn{9}{|c|}{ Panel B: Consider political risk in the models } \\
\hline LnGLOB & $\begin{array}{c}-5.15^{* * *} \\
(1.01)\end{array}$ & $\begin{array}{l}-11.07^{* *} \\
(2.22)\end{array}$ & $\begin{array}{c}-2.73^{*} \\
(1.19)\end{array}$ & $\begin{array}{l}4.92^{* * *} \\
(1.37)\end{array}$ & $\begin{array}{c}-1.92^{* *} \\
(0.59)\end{array}$ & $\begin{array}{l}-0.39 \\
(2.27)\end{array}$ & $\begin{array}{l}-8.62 * * \\
(2.02)\end{array}$ & $\begin{array}{c}-8.69 * * * * \\
(1.50)\end{array}$ \\
\hline LnPR & $\begin{array}{l}5.04^{* *} \\
(1.96)\end{array}$ & & $\begin{array}{l}-3.05 \\
(1.97)\end{array}$ & & $\begin{array}{l}6.18^{*} \\
(3.17)\end{array}$ & & $\begin{array}{l}-1.20 \\
(2.36)\end{array}$ & \\
\hline LnGLOB* $^{*} \mathrm{LnPR}$ & & $\begin{array}{l}1.36^{* *} \\
(0.39)\end{array}$ & & $\begin{array}{c}-1.45^{* * *} \\
(0.22)\end{array}$ & & $\begin{array}{l}-0.09 \\
(0.47)\end{array}$ & & $\begin{array}{c}0.06 \\
(0.30)\end{array}$ \\
\hline LnGDP & $\begin{array}{l}0.80 * \\
(0.36)\end{array}$ & $\begin{array}{l}1.26^{* *} \\
(0.32)\end{array}$ & $\begin{array}{l}-0.49 \\
(0.28)\end{array}$ & $\begin{array}{c}-0.89 * * * \\
(0.18)\end{array}$ & $\begin{array}{c}0.39 \\
(0.44)\end{array}$ & $\begin{array}{c}0.08 \\
(0.41)\end{array}$ & $\begin{array}{l}1.71 * \\
(0.64)\end{array}$ & $\begin{array}{l}2.04^{* * *} \\
(0.29)\end{array}$ \\
\hline LnREER & $\begin{array}{l}-0.12 \\
(1.04)\end{array}$ & $\begin{array}{l}-0.98 \\
(0.62)\end{array}$ & $\begin{array}{l}-0.14 \\
(0.72)\end{array}$ & $\begin{array}{c}0.21 \\
(0.43)\end{array}$ & $\begin{array}{c}1.16 \\
(1.21)\end{array}$ & $\begin{array}{c}0.58 \\
(1.14)\end{array}$ & $\begin{array}{l}-5.35^{*} \\
(1.98)\end{array}$ & $\begin{array}{c}-6.14^{* * * *} \\
(0.96)\end{array}$ \\
\hline Constant & $\begin{array}{l}-7.05 \\
(8.86)\end{array}$ & $\begin{array}{c}15.45^{* *} \\
(3.36)\end{array}$ & $\begin{array}{c}25.40^{* * *} \\
(7.23)\end{array}$ & $\begin{array}{l}7.60 * * \\
(2.98)\end{array}$ & $\begin{array}{l}-27.72 * \\
(13.84)\end{array}$ & $\begin{array}{l}-2.29 \\
(5.00)\end{array}$ & $\begin{array}{l}52.04^{* *} \\
(17.37)\end{array}$ & $\begin{array}{c}47.88^{* * *} \\
(6.33)\end{array}$ \\
\hline
\end{tabular}


Table 12. The estimated results of the error-correction model for tourism service trade balance by the ARDL model when considering the composite risk.

\begin{tabular}{|c|c|c|c|c|c|c|c|c|}
\hline \multirow[b]{2}{*}{ Variables } & \multicolumn{2}{|c|}{ Overall Globalization } & \multicolumn{2}{|c|}{ Economic Globalization } & \multicolumn{2}{|c|}{ Social Globalization } & \multicolumn{2}{|c|}{ Political Globalization } \\
\hline & $\begin{array}{c}\text { ARDL } \\
(4,0,3,3,2)\end{array}$ & $\begin{array}{c}\text { ARDL } \\
(3,3,4,4,4)\end{array}$ & $\begin{array}{c}\text { ARDL } \\
(1,3,3,3,3)\end{array}$ & $\begin{array}{c}\text { ARDL } \\
(1,1,3,4,0)\end{array}$ & $\begin{array}{c}\text { ARDL } \\
(1,1,3,3,1)\end{array}$ & $\begin{array}{c}\text { ARDL } \\
(3,3,3,4,4)\end{array}$ & $\begin{array}{c}\text { ARDL } \\
(3,4,4,3,4)\end{array}$ & $\begin{array}{c}\text { ARDL } \\
(3,3,3,3,3)\end{array}$ \\
\hline $\mathrm{D}(\operatorname{LnGLOB})_{\mathrm{t}}$ & & $\begin{array}{c}-21.23^{* * *} \\
(3.38)\end{array}$ & $\begin{array}{l}1.81^{*} \\
(0.94)\end{array}$ & $\begin{array}{l}5.11^{* * *} \\
(1.20)\end{array}$ & $\begin{array}{c}0.42 \\
(0.42)\end{array}$ & $\begin{array}{l}-4.41 * \\
(1.83)\end{array}$ & $\begin{array}{c}-4.99 * * * \\
(0.91)\end{array}$ & $\begin{array}{l}-1.41 \\
(1.50)\end{array}$ \\
\hline $\mathrm{D}(\operatorname{LnGLOB})_{\mathrm{t}-1}$ & & $\begin{array}{c}18.43^{* * * *} \\
(2.80)\end{array}$ & $\begin{array}{c}-3.89 * * * \\
(0.90)\end{array}$ & & & $\begin{array}{l}6.66^{* *} \\
(1.84)\end{array}$ & $\begin{array}{c}5.49^{* * *} \\
(1.14)\end{array}$ & $\begin{array}{l}2.74 * \\
(1.49)\end{array}$ \\
\hline $\mathrm{D}(\operatorname{LnGLOB})_{\mathrm{t}-2}$ & & $\begin{array}{c}15.59^{* * *} \\
(2.31)\end{array}$ & $\begin{array}{c}-2.98^{* *} \\
(0.99)\end{array}$ & & & $\begin{array}{c}12.35^{* * *} \\
(1.89)\end{array}$ & $\begin{array}{c}6.04^{* * *} \\
(1.20)\end{array}$ & $\begin{array}{c}7.53^{* * *} \\
(1.92)\end{array}$ \\
\hline $\mathrm{D}(\operatorname{LnGLOB})_{\mathrm{t}-3}$ & & & & & & & $\begin{array}{l}1.53 \\
(0.98)\end{array}$ & \\
\hline $\mathrm{D}(\mathrm{LnCR})_{\mathrm{t}}$ & $\begin{array}{l}-2.10 * \\
(1.09)\end{array}$ & & $\begin{array}{c}-5.62^{* * *} \\
(1.05)\end{array}$ & & $\begin{array}{c}-3.82 * * * \\
(1.10)\end{array}$ & & $\begin{array}{l}-4.31^{* *} \\
(1.34)\end{array}$ & \\
\hline $\mathrm{D}(\mathrm{LnCR})_{\mathrm{t}-1}$ & $\begin{array}{c}1.74 \\
(1.21)\end{array}$ & & $\begin{array}{c}1.29 \\
(1.08)\end{array}$ & & $\begin{array}{l}2.20^{*} \\
(1.14)\end{array}$ & & $\begin{array}{l}2.70 * \\
(1.21)\end{array}$ & \\
\hline $\begin{array}{l}\mathrm{D}(\mathrm{LnCR})_{\mathrm{t}-2} \\
\mathrm{D}(\mathrm{LnCR})_{\mathrm{t}-3}\end{array}$ & $\begin{array}{c}-2.67^{* *} \\
(1.11)\end{array}$ & & $\begin{array}{l}-2.91^{* *} \\
(0.98)\end{array}$ & & $\begin{array}{c}-2.71 \text { *** } \\
(0.81)\end{array}$ & & $\begin{array}{c}-3.11^{* *} \\
(1.04) \\
-2.01 * \\
(0.89)\end{array}$ & \\
\hline $\mathrm{D}\left(\mathrm{LnGLOB}^{*} \operatorname{LnCR}\right)_{\mathrm{t}}$ & & $\begin{array}{l}2.04^{* * *} \\
(0.44)\end{array}$ & & $\begin{array}{c}-1.18^{* * *} \\
(0.23)\end{array}$ & & $\begin{array}{c}0.37 \\
(0.35)\end{array}$ & & $\begin{array}{l}-0.61 * \\
(0.33)\end{array}$ \\
\hline $\mathrm{D}\left(\operatorname{LnGLOB}{ }^{*} \operatorname{LnCR}\right)_{\mathrm{t}-1}$ & & $\begin{array}{c}-1.52^{* * *} \\
(0.28)\end{array}$ & & $\begin{array}{c}-0.51^{* * *} \\
(0.14)\end{array}$ & & $\begin{array}{c}-0.99 * * \\
(0.35)\end{array}$ & & $\begin{array}{l}0.75^{* *} \\
(0.23)\end{array}$ \\
\hline $\mathrm{D}\left(\operatorname{LnGLOB}^{*} \operatorname{LnCR}\right)_{\mathrm{t}-2}$ & & $\begin{array}{c}-2.50^{* * *} \\
(0.32)\end{array}$ & & $\begin{array}{c}-1.18^{* * *} \\
(0.16)\end{array}$ & & $\begin{array}{c}-2.70^{* * *} \\
(0.40)\end{array}$ & & $\begin{array}{c}-0.74^{* * *} \\
(0.22)\end{array}$ \\
\hline $\mathrm{D}\left(\mathrm{LnGLOB}^{*} \operatorname{LnCR}\right)_{\mathrm{t}-3}$ & & $\begin{array}{c}0.96^{* * * *} \\
(0.16)\end{array}$ & & & & & & \\
\hline $\operatorname{ECT}(-1)$ & $\begin{array}{c}-0.86^{* * *} \\
(0.15)\end{array}$ & $\begin{array}{c}-1.88^{* * *} \\
(0.27)\end{array}$ & $\begin{array}{c}-0.56^{* * *} \\
(0.09)\end{array}$ & $\begin{array}{c}-0.47^{* * *} \\
(0.06)\end{array}$ & $\begin{array}{c}-0.46^{* * *} \\
(0.08)\end{array}$ & $\begin{array}{c}-1.42^{* * *} \\
(0.21)\end{array}$ & $\begin{array}{c}-1.65^{* * *} \\
(0.18)\end{array}$ & $\begin{array}{c}-1.42^{* * *} \\
(0.17)\end{array}$ \\
\hline
\end{tabular}

Notes: Standard errors are in parentheses. ${ }^{* * *},{ }^{* *}$, and ${ }^{*}$ indicate that the parameters are significant at the $1 \%, 5 \%$, and $10 \%$ levels, respectively. To save space, we only report the short-run coefficients of globalization, country risks, and ECT $(-1)$. 
Table 13. The estimated results of the error-correction model for tourism service trade balance by the ARDL model when considering the political risk.

\begin{tabular}{|c|c|c|c|c|c|c|c|c|}
\hline \multirow{2}{*}{ Variables } & \multicolumn{2}{|c|}{ Overall Globalization } & \multicolumn{2}{|c|}{ Economic Globalization } & \multicolumn{2}{|c|}{ Social Globalization } & \multicolumn{2}{|c|}{ Political Globalization } \\
\hline & $\begin{array}{c}\text { ARDL } \\
(3,2,3,4,4)\end{array}$ & $\begin{array}{c}\text { ARDL } \\
(4,4,4,4,4)\end{array}$ & $\begin{array}{c}\text { ARDL } \\
(4,3,4,4,0)\end{array}$ & $\begin{array}{c}\text { ARDL } \\
(\mathbf{4 , 0 , 4 , 4 , 0 )}\end{array}$ & $\begin{array}{c}\text { ARDL } \\
(3,2,3,4,4)\end{array}$ & $\begin{array}{c}\text { ARDL } \\
(3,0,2,3,1)\end{array}$ & $\begin{array}{c}\text { ARDL } \\
(4,4,4,3,4)\end{array}$ & $\begin{array}{c}\text { ARDL } \\
(1,3,3,3,2)\end{array}$ \\
\hline $\mathrm{D}(\operatorname{LnGLOB})_{\mathrm{t}}$ & $\begin{array}{c}-5.73^{* * *} \\
(0.98)\end{array}$ & $\begin{array}{c}-9.25^{* * *} \\
(1.51)\end{array}$ & $\begin{array}{l}-1.25 \\
(0.88)\end{array}$ & & $\begin{array}{c}-2.19 * * * \\
(0.48)\end{array}$ & & $\begin{array}{c}-6.78^{* * *} \\
(1.22)\end{array}$ & $\begin{array}{l}-2.48^{* *} \\
(1.10)\end{array}$ \\
\hline $\mathrm{D}(\operatorname{LnGLOB})_{\mathrm{t}-1}$ & $\begin{array}{l}3.45^{* *} \\
(1.06)\end{array}$ & $\begin{array}{c}16.38^{* * * *} \\
(2.50)\end{array}$ & $\begin{array}{c}0.04 \\
(0.74)\end{array}$ & & $\begin{array}{l}1.01^{* *} \\
(0.43)\end{array}$ & & $\begin{array}{l}4.61^{* *} \\
(1.35)\end{array}$ & $\begin{array}{l}4.96^{* * *} \\
(1.40)\end{array}$ \\
\hline $\mathrm{D}(\operatorname{LnGLOB})_{\mathrm{t}-2}$ & & $\begin{array}{c}15.90 * * * \\
(2.55)\end{array}$ & $\begin{array}{c}-4.50^{* * *} \\
(0.86)\end{array}$ & & & & $\begin{array}{l}4.11^{* *} \\
(1.39)\end{array}$ & $\begin{array}{l}4.02 * * \\
(1.45)\end{array}$ \\
\hline $\mathrm{D}(\operatorname{LnGLOB})_{\mathrm{t}-3}$ & & $\begin{array}{l}7.52^{* *} \\
(1.75)\end{array}$ & & & & & $\begin{array}{l}2.80 * \\
(1.04)\end{array}$ & \\
\hline $\mathrm{D}(\mathrm{LnPR})_{\mathrm{t}}$ & $\begin{array}{l}-0.46 \\
(0.97)\end{array}$ & & $\begin{array}{c}-5.35^{* * *} \\
(0.95)\end{array}$ & & $\begin{array}{l}-0.30 \\
(1.06)\end{array}$ & & $\begin{array}{l}-2.59 * \\
(1.08)\end{array}$ & \\
\hline $\mathrm{D}(\mathrm{LnPR})_{\mathrm{t}-1}$ & $\begin{array}{c}-1.67 * \\
(0.82)\end{array}$ & & $\begin{array}{c}-3.54^{* * *} \\
(0.87)\end{array}$ & & $\begin{array}{l}-2.01 \text { * } \\
(0.96)\end{array}$ & & $\begin{array}{l}2.24 * \\
(1.02)\end{array}$ & \\
\hline $\mathrm{D}(\mathrm{LnPR})_{\mathrm{t}-2}$ & $\begin{array}{c}-6.05^{* * *} \\
(1.00)\end{array}$ & & $\begin{array}{c}-5.54^{* * *} \\
(0.89)\end{array}$ & & $\begin{array}{c}-6.02 * * * \\
(1.17)\end{array}$ & & $\begin{array}{l}-2.50 * \\
(1.03)\end{array}$ & \\
\hline $\mathrm{D}(\mathrm{LnPR})_{\mathrm{t}-3}$ & & & $\begin{array}{c}-1.41^{* *} \\
(0.58)\end{array}$ & & & & $\begin{array}{l}-1.43 \\
(0.90)\end{array}$ & \\
\hline $\mathrm{D}\left(\mathrm{LnGLOB}^{*} \mathrm{LnPR}\right)_{\mathrm{t}}$ & & $\begin{array}{c}0.48 \\
(0.26)\end{array}$ & & $\begin{array}{c}-1.77^{* * *} \\
(0.23)\end{array}$ & & $\begin{array}{l}0.20 * \\
(0.11)\end{array}$ & & $\begin{array}{l}-0.12 \\
(0.21)\end{array}$ \\
\hline $\mathrm{D}\left(\operatorname{LnGLOB}^{*} \operatorname{LnPR}\right)_{\mathrm{t}-1}$ & & $\begin{array}{c}-1.38^{* *} \\
(0.30)\end{array}$ & & $\begin{array}{c}-0.59 * * * \\
(0.14)\end{array}$ & & $\begin{array}{c}0.50^{* * * *} \\
(0.11)\end{array}$ & & $\begin{array}{c}0.57^{* * *} \\
(0.16)\end{array}$ \\
\hline $\mathrm{D}\left(\mathrm{LnGLOB}{ }^{*} \operatorname{LnPR}\right)_{\mathrm{t}-2}$ & & $\begin{array}{c}-1.85^{* * *} \\
(0.25)\end{array}$ & & $\begin{array}{c}-1.35^{* * *} \\
(0.18)\end{array}$ & & & & $\begin{array}{c}-0.36^{*} \\
(0.18)\end{array}$ \\
\hline $\mathrm{D}\left(\mathrm{LnGLOB}^{*} \operatorname{LnPR}\right)_{\mathrm{t}-3}$ & & $\begin{array}{c}-0.58 \text { * } \\
(0.22)\end{array}$ & & $\begin{array}{l}-0.34^{* *} \\
(0.13)\end{array}$ & & & & \\
\hline $\mathrm{ECT}(-1)$ & $\begin{array}{c}-1.39 * * * \\
(0.20)\end{array}$ & $\begin{array}{c}-2.97^{* * *} \\
(0.40)\end{array}$ & $\begin{array}{c}-1.46^{* * *} \\
(0.17)\end{array}$ & $\begin{array}{c}-1.47^{* * *} \\
(0.19)\end{array}$ & $\begin{array}{c}-1.20 * * * \\
(0.20)\end{array}$ & $\begin{array}{c}-0.99 * * * \\
(0.18)\end{array}$ & $\begin{array}{c}-1.51^{* * *} \\
(0.19)\end{array}$ & $\begin{array}{c}-1.36^{* * *} \\
(0.19)\end{array}$ \\
\hline
\end{tabular}

Notes: Standard errors are in parentheses. ${ }^{* * *},{ }^{* *}$, and ${ }^{*}$ indicate that the parameters are significant at the $1 \%, 5 \%$, and $10 \%$ levels, respectively. To save space, we only report the short-run coefficients of globalization, country risks, and ECT $(-1)$. 


\subsection{The CUSUM and CUSUMSQ Tests of Stability}

We applied the cumulative sum (CUSUM) of recursive residuals and the CUSUM of square (CUSUMSQ) tests to assess parameter stability. The test results show that plots of the CUSUM and CUSUMSQ statistics fall inside the critical bands of the $5 \%$ confidence intervals of parameter stability, indicating the absence of any instability of these coefficients. To save space, this study does not report the results of CUSUM and CUSUMSQ tests.

\section{Discussion and Implication}

Tourism and its associated economic repercussions have taken place within a wider context of globalization of the world economy [44]. It is thus logical to consider tourism as a main component in the process of globalization [14], but trade in tourism services and the benefits of free trade in these services are topics that have received less attention in international economics. This study looks to provide an empirical explanation for the relationships between tourism service trade, globalization, and country risks in China by using the ARDL model.

In the long run, we find that globalization has negative effects on the exports of tourism service trade, but positive effects on its imports, thus exacerbating the tourism service trade imbalances. The positive impact of globalization on tourism service imports found in our study is roughly consistent with the findings of Javid and Katircioglu [23] for a sample of 133 countries and of Ivanov and Webster [10] for 167 countries. However, both of them also found a positive impact of globalization on tourism service exports, which is completely opposite to our findings. This indicates that compared with other countries, the impact of globalization on China's tourism service trade has certain characteristics. The auses for this may be as follows. First, with the development of information and communications technology (social globalization), tourists could easily gather information on destinations, products, and services, which expands their choices $[69,70]$. The international competitiveness of China's tourism service trade is low [8]. Tourism infrastructure, products, and services of the country cannot meet the needs of the international tourism market, thus inhibiting the exports of tourism service trade.

Second, the globalization of liberalization of trade (economic globalization) creates new markets and new sources of competition for the tourism industry [15]. Some countries provide characteristic tourism services and products to expand the market. For instance, South Korea specifically attracts high-end Chinese female consumers through department stores, shopping outlets, and some famous attractions [8]. Furthermore, some countries also have succeeded in attracting Chinese tourists by adopting clear tourism development policies, such as easier visa facilitation (political globalization), shopping tax exemption (economic globalization), the opening of air rights, low-cost routes (social globalization), etc. [71]. Therefore, the choice of a large number of Chinese tourists to travel abroad increases the country's tourism service trade imports.

Some implications did arise from our findings. In the area of policy, knowledge of the facts herein can assist regulators and policy makers in determining and executing suitable effective policies. First, the government should promote sound economic policies and good governance, which can help build buffers against the adverse effects of globalization on tourism service exports and trade balances. Second, the government should fully exploit the advantages of tourism resources and build a multinational boutique tourism route system. Third, the government should continue to promote easier visa facilitation and a tax refund policy for shopping by foreign tourists. Fourth, in respect of social globalization, the government should also strengthen online interactive marketing and build an "Internet +" overseas tourism promotion system. Fifth, in terms of country risks, the government needs to maintain the stability of country environments since the role of country risks in moderating the relationship between globalization and tourism service trade is important. At the same time, they should improve their ability to quickly deal with earth-shattering events such as COVID-19, so as to ensure the sustainable development of tourism service trade after being shocked.

The limitation of this study is that we adopted aggregated data, which could not consider the differences between the effects for the individual tourism services trading partners, bringing about 
aggregation bias. In the future, we could adopt disaggregated data, i.e., the bilateral tourism services trade, to explore the effects of globalization on the bilateral tourism services trade (i.e., export, import, and trade balance) for China and other individual countries.

Author Contributions: Formal analysis, writing—review \& editing, W.Z. and Y.-B.C.; conceptualization, data curation, supervision, and validation, Y.-B.C.; methodology, writing-original draft preparation, W.Z. All authors have read and agreed to the published version of the manuscript.

Funding: This research was supported by the National Natural Science Foundation of China (grant number 71771188).

Conflicts of Interest: The authors declare no conflict of interest.

\section{References}

1. Hidayati, F. Tourism and economic growth: The role of globalization. J. Public Adm. Stud. 2018, 2, 16-20.

2. Lin, V.S.; Yang, Y.; Li, G. Where can tourism-led growth and economy-driven tourism growth occur. J. Travel Res. 2019, 58, 760-773. [CrossRef]

3. Narayan, P.K.; Narayan, S.; Prasad, A.; Prasad, B.C. Tourism and economic growth: A panel data analysis for Pacific Island countries. Tour. Econ. 2010, 16, 169-183. [CrossRef]

4. Tang, C.F.; Tan, E.C. Does tourism effectively stimulate Malaysia's economic growth. Tour. Manag. 2015, 46, 158-163. [CrossRef]

5. Fayed, H.; Fletcher, J. Report: Globalization of economic activity: Issues for tourism. Tour. Econ. 2002, 8, 207-230. [CrossRef]

6. Kumar, M.; Prashar, S.; Jana, R.K. Does international tourism spur international trade and output. Evidence from wavelet analysis. Tour. Econ. 2019, 25, 22-33. [CrossRef]

7. Li, Y. Difficulties and Countermeasures to Improving of Inbound Tourism in China. In Proceedings of the 4th International Conference on Education Reform and Modern Management, Phuket, Thailand, 6-7 August 2017.

8. Guo, C.; Liao, C. Research on the Deficit of China's Tourism Service Trade and Countermeasures. Am. J. Ind. Bus. Manag. 2017, 7, 170-178. [CrossRef]

9. Dreher, A. Does globalization affect growth. Evidence from a new index of globalization. Appl. Econ. 2006, 38, 1091-1110. [CrossRef]

10. Ivanov, S.H.; Webster, C. Tourism's impact on growth: The role of globalisation. Ann. Tour. Res. 2013, 41, 231-236. [CrossRef]

11. Song, H.; Li, G.; Cao, Z. Tourism and Economic Globalization: An emerging research agenda. J. Travel Res. 2018, 57, 999-1011. [CrossRef]

12. Chiu, Y.-B.; Zhang, W.; Ding, K. Does globalization influence inbound tourism. Evidence from a dynamic panel threshold analysis. J. Travel Res. 2020. [CrossRef]

13. Hociung, I.G.; Frâncu, L.G. Globalization-tourism-communication, competitiveness triangle on the market affected by the economic crisis. Theor. Appl. Econ. 2012, 19, 133-146.

14. Mustafa, M.H. Tourism and globalization in the Arab world. Int. J. Bus. Soc. Sci. 2010, 1, 37-48.

15. Dwyer, L. Globalization of tourism: Drivers and outcomes. Tour. Recreat Res. 2015, 40, 326-339. [CrossRef]

16. Jin, X.; Qu, M.; Bao, J. Impact of crisis events on Chinese outbound tourist flow: A framework for post-events growth. Tour. Manag. 2019, 74, 334-344. [CrossRef]

17. Brahmasrene, T.; Lee, J.W. Assessing the dynamic impact of tourism, industrialization, urbanization, and globalization on growth and environment in Southeast Asia. Int. J. Sustain. Dev. World Ecol. 2017, 24, 362-371. [CrossRef]

18. Saint Akadiri, S.; Lasisi, T.T.; Uzuner, G.; Akadiri, A.C. Examining the impact of globalization in the environmental Kuznets curve hypothesis: The case of tourist destination states. Environ. Sci. Pollut. Res. 2019, 26, 12605-12615. [CrossRef]

19. Kulczyk-Dynowska, A.; Bal-Domańska, B. The National parks in the context of tourist function development in territorially linked municipalities in poland. Sustainability 2019, 11, 1996. [CrossRef]

20. Floyd, M.F.; Pennington-Gray, L. Profiling risk perceptions of tourists. Ann. Tour. Res. 2004, 31, 1051-1054. [CrossRef] 
21. Wang, J.; Liu-Lastres, B.; Ritchie, B.W.; Pan, D.Z. Risk reduction and adventure tourism safety: An extension of the risk perception attitude framework (RPAF). Tour. Manag. 2019, 74, 247-257. [CrossRef]

22. Drakos, K.; Kutan, A.M. Regional Effects of Terrorism on Tourism in Three Mediterranean Countries. J. Confl. Resolut. 2003, 47, 621-641. [CrossRef]

23. Javid, E.; Katircioglu, S. The globalization indicators-tourism development nexus: A dynamic panel-data analysis. Asia Pac. J. Tour. Res. 2017, 22, 1194-1205. [CrossRef]

24. Bakar, N.A.; Rosbi, S. Effect of Coronavirus disease (COVID-19) to tourism industry. Int. J. Adv. Eng. Res. Sci. 2020, 7, 189-193. [CrossRef]

25. Chang, C.L.; McAleer, M.; Ramos, V. A charter for sustainable tourism after COVID-19. Sustainability 2020, 12, 3671. [CrossRef]

26. World Tourism Organization. Impact Assessment of the COVID-19 Outbreak on International Tourism; World Tourism Organization: Madrid, Spain, 2020.

27. Moore, M. APAC: COVID-19 Impact on Tourist Arrivals by Country or Region; Statista: Hamburg, Germany, 2020. Available online: https://www.statista.com/statistics/1103147/apac-covid-19-impact-on-tourist-arrivals-bycountry/ (accessed on 10 June 2020).

28. Cheer, J.M. Human flourishing, tourism transformation and COVID-19: A conceptual touchstone. Tour. Geogr. 2020. [CrossRef]

29. Gössling, S.; Scott, D.; Hall, C.M. Pandemics, tourism and global change: A rapid assessment of COVID-19. J. Sustain. Tour. 2020. [CrossRef]

30. Hall, C.M.; Scott, D.; Gössling, S. Pandemics, transformations and tourism: Be careful what you wish for. Tour. Geogr. 2020. [CrossRef]

31. Hjalager, A.M. Stages in the economic globalization of tourism. Ann. Tour. Res. 2007, 34, 437-457. [CrossRef]

32. Prideaux, B. Factors affecting bilateral tourism flows. Ann. Tour. Res. 2005, 32, 780-801. [CrossRef]

33. Vietze, C. Cultural effects on inbound tourism into the USA: A gravity approach. Tour. Econ. 2012, 18, 121-138. [CrossRef]

34. Kim, S.S.; Prideaux, B.; Timothy, D. Factors affecting bilateral Chinese and Japanese travel. Ann. Tour. Res. 2016, 61, 80-95.

35. Harb, G.; Bassil, C. Gravity analysis of tourism flows and the 'multilateral resistance to tourism'. Curr. Issues Tour. 2020, 23, 666-678. [CrossRef]

36. Dai, B.; Jiang, Y.; Yang, L.; Ma, Y. China's outbound tourism-Stages, policies and choices. Tour. Manag. 2017, 58, 253-258. [CrossRef]

37. Read, R. The implications of increasing globalization and regionalism for the economic growth of small island states. World Dev. 2004, 32, 365-378. [CrossRef]

38. Nyaupane, G.P.; Timothy, D.J. Power, regionalism and tourism policy in Bhutan. Ann. Tour. Res. 2010, 37, 969-988. [CrossRef]

39. Henderson, J.C. Regionlisation and tourism: The Indonesia-Malaysia-Singapore growth triangle. Curr. Issues Tour. 2001, 4, 78-93. [CrossRef]

40. Dredge, D.; Jenkins, J. Destination place identity and regional tourism policy. Tour. Geogr. 2003, 5, $383-407$. [CrossRef]

41. Johnson, C.; Vanetti, M. Locational strategies of international hotel chains. Ann. Tour. Res. 2005, 32, 1077-1099. [CrossRef]

42. Rogerson, J.M. Unpacking the growth of hotel chains in Africa: Enterprises and patterns. Mediterr. J. Soc. Sci. 2014, 5, 135-146. [CrossRef]

43. Clancy, M. Global commodity chains and tourism: Past research and future directions. In Political Economy of Tourism: A Critical Perspective; Routledge: London, UK, 2010; pp. 99-116.

44. Rey, B.; Myro, R.L.; Galera, A. Effect of low-cost airlines on tourism in Spain. A dynamic panel data model. J. Air Transp. Manag. 2011, 17, 163-167. [CrossRef]

45. Sugiyarto, G.; Blake, A.; Sinclair, M.T. Tourism and globalization: Economic impact in Indonesia. Ann. Tour. Res. 2003, 30, 683-701. [CrossRef]

46. Stezhko, N.; Oliinyk, Y.; Polishchuk, L.; Tyshchuk, I.; Parfinenko, A.; Markhonos, S. International Tourism in the System of Modern Globalization Processes. Int. J. Manag. 2020, 11, 97-106.

47. Sequeira, T.; Nunes, P.M. Does tourism influence economic growth. A dynamic panel data approach. Econ. Rec. 2008, 84, 223-236. [CrossRef] 
48. Scheyvens, R. The challenge of sustainable tourism development in the Maldives: Understanding the social and political dimensions of sustainability. Asia Pac. Viewp. 2011, 52, 148-164. [CrossRef]

49. Eilat, Y.; Einav, L. Determinants of international tourism: A three-dimensional panel data analysis. Appl. Econ. 2004, 36, 1315-1327. [CrossRef]

50. Yap, G.; Saha, S. Do political instability, terrorism, and corruption have deterring effects on tourism development even in the presence of UNESCO heritage. A cross-country panel estimate. Tour. Anal. 2013, 18, 587-599. [CrossRef]

51. Joshi, O.; Poudyal, N.C.; Larson, L.R. The influence of sociopolitical, natural, and cultural factors on international tourism growth: A cross-country panel analysis. Environ. Dev. Sustain. 2017, 19, 825-838. [CrossRef]

52. Saha, S.; Su, J.-J.; Campbell, N. Does Political and Economic Freedom Matter for Inbound Tourism? A Cross-National Panel Data Estimation. J. Travel Res. 2017, 56, 221-234. [CrossRef]

53. Musavengane, R.; Siakwah, P.; Leonard, L. The nexus between tourism and urban risk: Towards inclusive, safe, resilient and sustainable outdoor tourism in African cities. J. Outdoor Recreat. Tour. 2020, 29, 100254. [CrossRef]

54. Held, D.; McGrew, A.; Goldblatt, D.; Perraton, J. Global Transformations: Politics, Economics and Culture; Stanford University Press: Stanford, CA, USA, 2000.

55. Ivanov, S.H.; Ivanova, M.G. Do hotel chains improve destination's competitiveness. Tour. Manag. Perspect. 2016, 19, 74-79. [CrossRef]

56. Alola, U.V.; Cop, S.; Alola, A.A. The spillover effects of tourism receipts, political risk, real exchange rate, and trade indicators in Turkey. Int. J. Tour. Res. 2019, 21, 813-823. [CrossRef]

57. Pesaran, M.H.; Shin, Y.; Smith, R. Bounds testing approaches to the analysis of level relationships. Appl. Econom. 2001, 16, 289-326. [CrossRef]

58. Dreher, A.; Gaston, N.; Martens, P. Measuring Globalization-Gauging Its Consequences; Springer: New York, NY, USA, 2008.

59. Dickey, D.A.; Fuller, W.A. Distribution of the estimation for autoregressive time series with a unit root. J. Am. Stat. Assoc. 1979, 74, 427-431.

60. Phillips, P.C.B.; Perron, P. Testing for a Unit root in Time Series Regression. Biometrika 1988, 75, 335-346. [CrossRef]

61. Johansen, S.; Juselius, K. Maximum likelihood estimation and inference on cointegration with applications to the demand for money. Oxf. Bull. Econ. Stat. 1990, 52, 161-210. [CrossRef]

62. Berrittella, M.; Bigano, A.; Roson, R.; Tol, R.S. A general equilibrium analysis of climate change impacts on tourism. Tour. Manag. 2006, 27, 913-924. [CrossRef]

63. Massidda, C.; Mattana, P. A SVECM analysis of the relationship between international tourism arrivals, GDP and trade in Italy. J. Travel Res. 2013, 52, 93-105. [CrossRef]

64. Dritsakis, N. Tourism as a long-run economic growth factor: An empirical investigation for Greece using causality analysis. Tour. Econ. 2004, 10, 305-316. [CrossRef]

65. Kunst, I. The role of the government in promoting tourism investment in selected Mediterranean countries-implications for the Republic of Croatia. Tour. Hosp. Manag. 2011, 17, 115-130.

66. Wong, A. China's Current Account: External Rebalancing or Capital Flight. Int. Financ. Discuss. Pap. 2017. [CrossRef]

67. Kwack, S.Y.; Ahn, C.Y.; Lee, Y.S.; Yang, D.Y. Consistent estimates of world trade elasticities and an application to the effects of Chinese Yuan (RMB) appreciation. J. Asian Econ. 2007, 18, 314-330. [CrossRef]

68. Dogru, T.; Isik, C.; Sirakaya-Turk, E. The balance of trade and exchange rates: Theory and contemporary evidence from tourism. Tour. Manag. 2019, 74, 12-23. [CrossRef]

69. Zheng, X.; Magnini, V.P.; Fesenmaier, D.R. Information technology and consumer behavior in travel and tourism: Insights from travel planning using the internet. J. Retail. Consum. Serv. 2015, 22, 244-249.

70. Zheng, X. From digitization to the age of acceleration: On information technology and tourism. Tour. Manag. Perspect. 2018, 25, 147-150.

71. Zhao, R.J. Analysis on the causes and countermeasures of China's tourism trade deficit. Chongqing Soc. Sci. 2016, 4, 19-25.

(C) 2020 by the authors. Licensee MDPI, Basel, Switzerland. This article is an open access article distributed under the terms and conditions of the Creative Commons Attribution (CC BY) license (http://creativecommons.org/licenses/by/4.0/). 